\title{
SELECTING REDUCED MODELS IN THE CROSS-ENTROPY METHOD
}

\author{
P. HÉAS *
}

\begin{abstract}
This paper deals with the estimation of rare event probabilities using importance sampling (IS), where an optimal proposal distribution is computed with the cross-entropy (CE) method. Although, IS optimized with the CE method leads to an efficient reduction of the estimator variance, this approach remains unaffordable for problems where the repeated evaluation of the score function represents a too intensive computational effort. This is often the case for score functions related to the solution of a partial differential equation (PDE) with random inputs. This work proposes to alleviate computation by the parsimonious use of a hierarchy of score function approximations in the CE optimization process. The score function approximation is obtained by selecting the surrogate of lowest dimensionality, whose accuracy guarantees to pass the current CE optimization stage. The selection relies on certified upper bounds on the error norm. An asymptotic analysis provides some theoretical guarantees on the efficiency and convergence of the proposed algorithm. Numerical results demonstrate the gain brought by the method in the context of pollution alerts and a system modeled by a PDE.
\end{abstract}

Key words. Rare event simulation, importance sampling, cross-entropy optimization, reduced basis, certified error bound, partial differential equation.

1. Introduction. The accurate estimation of the probability of a rare event with standard Monte Carlo typically requires the evaluation of a score function for a very large set of points: the number of points is of the order of the inverse of the sought rare event probability. The evaluation of the score function for this very large set becomes particularly infeasible if for each point a computationally expensive model is involved.

IS is a variance reduction strategy for Monte Carlo estimation. The idea is to sample from a biasing distribution, such that fewer samples are necessary to obtain the same accuracy of the rare event probability than with standard Monte Carlo. The bias introduced by the sampling from the biasing distribution is corrected by reweighing the samples in the IS estimator.

The key of the performance of IS lies in the choice of the problem-dependent so-called biasing or proposal distribution. Although the optimal biasing distribution that leads to a zero variance IS estimator is closed-form, computing this zero-variance density is not straightforward since it requires the sought probability of the rare event. The $\mathrm{CE}$ method [1, 2, 3, 4, provides an efficient way to approximate this zero-variance density within a parametric family of probability distributions. The CE method searches the optimal biasing distribution, in the sense that it will minimise the Kullback-Leibler divergence from the zero-variance density among the feasible distributions. Even though, computing the biasing distribution with the CE method may still be prohibitive if the model involved in the score function is computationally demanding.

This paper is concerned by the following question: can we save computational power by using score function approximations in the CE method? Several works recommend score function approximations in order to accelerate the estimation of rare event probabilities. In particular, score function approximations have already been put forward in IS [5, 6, 7, 8, 9, $]^{1}$ Nevertheless, to the best of our knowledge,

*INRIA \& IRMAR, Univ Rennes, Campus de Beaulieu, 35042 Rennes, France. (patrick.heas@inria.fr).

${ }^{1}$ We note that such approximations have also been studied in the context of multilevel split- 
the methods proposed in [12] and [13] are the unique works directly concerned by the raised question and providing partial answers. The authors in [12] define a sequence of auxiliary "low-fidelity CE methods" using a set of score function approximations. The auxiliary low-fidelity CE methods are then run sequentially in order to pre-condition the standard CE method defined with the original score function. Under certain conditions on score function approximations, the pre-conditioning guarantees that the number of iterations is lowered at each of the auxiliary CE method levels, or at worst remains identical. Nevertheless, using this pre-conditioner does not necessarily guarantee a reduction of the global computational cost for computing the biasing distribution. The pre-conditioner is in fact an initialization strategy rather than a way of integrating and adjusting the score function approximations in the CE method. Computational power may still be spoilt in the case an accurate estimate is not needed at some levels of the pre-conditioning sequence. In [13, the authors propose an IS estimator based on an hybrid "low-fidelity / high-fidelity CE method". The idea is to approximate the score function, by dividing the probability space into two subdomains. Regions which are close in some sense to the rare event are evaluated with the high-fidelity model. The remaining part of the probability space is evaluated using a low-cost surrogate model. However, parameters of the proposed algorithms are exposed to arbitrariness, leading to non-certified sub-domain discrimination, which in turns implies the computation of a sub-optimal biasing distribution.

In this work, we propose a $\mathrm{CE}$ algorithm based on score function approximations which converges almost surely to the optimal biasing distribution. A surrogate is selected in a hierarchy of precomputed score function approximations at each iteration of the $\mathrm{CE}$ method, depending on the needed accuracy. As for the certified reduced-basis evaluation of failure probability in 14, the selection of the score function approximations exploits upper bounds on the error norm of the reduced model. We focus on reduced basis $(\mathrm{RB})$ approximations, for which there exists an extensive literature on the computation of a priori or a posteriori error estimates for approximation of the solution of a PDE using RB, see e.g., [15. Besides, we also provide an asymptotic analysis showing that under mild conditions i) for each of the algorithm iterations, the squared coefficient of variation (SCV) of the IS estimator is the minimal achievable with the current score function approximation, ii) the convergence is guaranteed towards the optimal biasing distribution in at most the same number of iterations as the standard CE method.

The paper is organized as follows. Section 2 recalls the basics of IS for rare event estimation with the $\mathrm{CE}$ method. It then introduces score function approximations and reduced models. In Section 3 , we present state-of-the-art approaches, and in particular the method pre-conditioning the CE method. We then propose in Section 4 our algorithm. A theoretical result attesting of the asymptotic performance of our method is given in Section 5. We provide details on the proof of the proposed theorem in the appendices. Section 6 presents the numerical evaluation in the case of rare event probability estimation related to a pollution alert problem. We finally provide concluding remarks in a last section.

2. Efficient Rare Event Estimation by Importance Sampling. We assume that $X$ is a random element taking its values in $\mathbb{R}^{p}$ and denote by $\mu$ its probability

ting [10, 11]. However, an exhaustive discussion of all possible combinations of alternative estimation techniques with score function approximations is out of the scope of this work. 
distribution.We denote by $A$ the set of rare events of interest, and we assume that

$$
A=\left\{x \in \mathbb{R}^{p}: \phi(x) \geq \gamma^{\star}\right\},
$$

for some real number $\gamma^{\star}$ and for a score function $\phi: \mathbb{R}^{p} \rightarrow \mathbb{R}$. The probability of the rare event $X \in A$ is defined as $p_{A}=\left\langle\mathbb{1}_{A}, \mu\right\rangle$, where $\langle\cdot, \mu\rangle$ denotes an integration with respect to the probability measure $\mu$. We look for an estimator of $p_{A}$ where $\gamma^{\star}$ is large so that $p_{A} \ll 1$. We assume that we know how to draw $m$ independent and identically distributed (i.i.d.) samples $x_{1}, \cdots, x_{m}$ from $\mu$.

2.1. First Ingredient: the Cross-Entropy Method. The naive Monte-Carlo (MC) estimator of the rare event probability is

$$
p_{A}^{M C}=\frac{1}{m} \sum_{i=1}^{m} \mathbb{1}_{A}\left(x_{i}\right) .
$$

This estimator is unbiased. Its relative error is measured by the SCV of the estimator, which is

$$
\frac{\mathbb{E}\left[\left(p_{A}^{M C}-p_{A}\right)^{2}\right]}{p_{A}^{2}}=\frac{1}{m}\left(\frac{1}{p_{A}}-1\right) \stackrel{p_{A} \ll 1}{\simeq} \frac{1}{m p_{A}}
$$

so that we need $m>p_{A}^{-1}$ for a relative error smaller than one.

Let $\operatorname{supp}(\mu)=\left\{x \in \mathbb{R}^{p}: \mu(x)>0\right\}$ be the support of the distribution $\mu$. For a biasing distribution $\nu$ with $\operatorname{supp}(\mu) \subseteq \operatorname{supp}(\nu)$, the IS estimator $p_{A, \nu}^{I S}$ of $p_{A}$ is

$$
p_{A, \nu}^{I S}=\frac{1}{m} \sum_{i=1}^{m} \mathbb{1}_{A}\left(z_{i}\right) \frac{\mu\left(z_{i}\right)}{\nu\left(z_{i}\right)}
$$

with $m$ i.i.d. samples $z_{1}, \cdots, z_{m}$ from $\nu$. It is easy to see that $p_{A, \nu}^{I S}$ is an unbiased estimator of $p_{A}$. Its $\mathrm{SCV}$ is

$$
\frac{\mathbb{E}\left[\left(p_{A, \nu}^{I S}-p_{A}\right)^{2}\right]}{p_{A}^{2}}=\frac{1}{m p_{A}^{2}} \operatorname{var}\left(\mathbb{1}_{A} \frac{\mu}{\nu}, \nu\right) .
$$

The optimal biasing distribution $\nu_{A}^{\star}$ yielding a zero-variance estimator (i.e., a zero $\mathrm{SCV})$ is

$$
\nu_{A}^{\star}=\frac{\mathbb{1}_{A} \mu}{p_{A}} .
$$

Unfortunately, it depends on the rare event probability $p_{A}$ we want to estimate. Now, consider a set of parametrized densities $\mathcal{V}=\left\{\nu^{\theta}: \theta \in \Theta\right\}$. The CE method [1] optimizes for a parameter $\theta^{\star} \in \Theta$ such that the corresponding distribution $\nu_{A}^{\theta^{\star}}$ minimizes the Kullback-Leibler divergence from the zero-variance density $\nu_{A}^{\star}$ :

$$
\nu_{A}^{\theta^{\star}} \in \underset{\nu^{\theta} \in \mathcal{V}}{\arg \min }\left\langle\ln \frac{\nu_{A}^{\star}}{\nu^{\theta}}, \nu_{A}^{\star}\right\rangle .
$$

In the desirable situation where $\nu_{A}^{\star} \in \mathcal{V}$, a minimizer $\theta^{\star}$ of 2.5 is such that $\nu_{A}^{\theta^{\star}}=\nu_{A}^{\star}$. Using 2.4, the optimization problem 2.5 can be rewritten as

$$
\nu_{A}^{\theta^{\star}} \in \underset{\nu^{\theta} \in \mathcal{V}}{\arg \max }\left\langle\mathbb{1}_{A} \ln \nu^{\theta}, \mu\right\rangle
$$


Solving the stochastic counterpart of 2.6

$$
\hat{\nu}_{A}^{\theta^{\star}} \in \underset{\nu^{\theta} \in \mathcal{V}}{\arg \max } \frac{1}{m} \sum_{i=1}^{m} \mathbb{1}_{A}\left(x_{i}\right) \ln \nu^{\theta}\left(x_{i}\right),
$$

with $m$ i.i.d. samples $x_{1}, \cdots, x_{m}$ from $\mu$, typically fails because (2.7) is affected by the rareness of the event $\mathbb{1}_{A}(X)=1$, just as for the naive MC estimator 2.1). To circumvent this effect, starting from the initial distribution $\nu_{0}=\mu$ and for some parameter $\rho \in(0,1)$, the $\mathrm{CE}$ method estimates sequentially a sequence of nested events

$$
A_{1} \supseteq A_{2} \supseteq \cdots \supseteq A,
$$

such that $A_{j+1}=\left\{x \in \mathbb{R}^{p}: \phi(x) \geq \gamma\left(\nu_{j}, \rho, \phi\right)\right\}$ with the $(1-\rho)$-quantile

$$
\gamma\left(\nu_{j}, \rho, \phi\right)=\max \left\{s \in \mathbb{R}:\left\langle\mathbb{1}_{\phi(\cdot) \geq s}, \nu_{j}\right\rangle \geq \rho\right\}=\max \left\{s \in \mathbb{R}:\left\langle\mathbb{1}_{\phi(\cdot)<s}, \nu_{j}\right\rangle \leq 1-\rho\right\},
$$

and jointly updates the biasing distribution $\nu_{j}$ according to

$$
\nu_{j} \in \underset{\nu^{\theta} \in \mathcal{V}}{\arg \max }\left\langle\mathbb{1}_{A_{j}} \frac{\mu}{\nu_{j-1}} \ln \nu^{\theta}, \nu_{j-1}\right\rangle .
$$

In words, the occurrence of the event $\mathbb{1}_{A_{j}}(X)=1$ where $X$ is a random variable of distribution $\nu_{j-1}$ tends on the one hand to decrease since we have $A_{j-1} \supseteq A_{j}$. On the other hand, it tends to increase since, according to (2.8) - 2.9), $\nu_{j}$ is nearer (in terms of cross-entropy) from $\nu_{A}^{\star}$ than $\nu_{j-1}$. Typically, a proper setting for this tradeoff will yield an occurrence of the event $\mathbb{1}_{A_{j}}(X)=1$ that varies little with the algorithm iterations. Then, if the set $A_{1}$ and the initial distribution are chosen so that the event $\mathbb{1}_{A_{1}}(X)=1$ is not rare, we can typically expect that the solution of the stochastic counterpart of problem (2.9) will be a "good" approximation of $\nu_{j}$, and that the CE method will yield a "good" approximation of $\nu_{A}^{\theta^{*}}$.

2.2. Second Ingredient: Score Function Approximations. We consider a high-fidelity model $f^{\star}: \mathbb{R}^{p} \rightarrow \mathbb{R}^{q}$ parametrized by $x \in \mathbb{R}^{p}$. Let $\mathcal{X} \subset \mathbb{R}^{p}$ be the parameter space. Furthermore, we assume that we have at our disposal a set of model evaluations $\left\{f^{\star}\left(x_{i}\right)\right\}_{i=1}^{N}$, so-called snapshots, corresponding to $N$ representative samples $x_{i}$ of the parameter space $\mathcal{X}$. We focus in this work on reduced modeling strategies, which uses the set of snapshots to construct an approximation subspace $V_{K}$ for the set $\left\{f^{\star}(x): x \in \mathcal{X}\right\}$. Typical examples of such strategies are the common greedy RB methods or principal orthogonal decomposition (POD). Those standard techniques usually generate the approximation subspace $V_{K}$ together with a sequence of nested RB spaces $V_{1} \subset V_{2} \subset \ldots \subset V_{K}$, of increasing dimension $d_{k}=\operatorname{dim}\left(V_{k}\right)$. A set $\left\{f^{(k)}\left(x_{i}\right)\right\}_{k=1}^{K}$ of low-dimensional approximations of the snapshot $f^{\star}\left(x_{i}\right)$, also called reduced models or surrogates, is then computable using the sequence of nested subspaces. For instance, $f^{\star}\left(x_{i}\right)$ can be a finite element discretization of a PDE and $f^{(k)}\left(x_{i}\right)$ some PetrovGalerkin or least-square RB approximation [15. We will adopt the following general notations: we assume available a hierarchy $\mathcal{F}=\left\{f^{(1)}, f^{(2)}, \ldots, f^{(K)}, f^{(K+1)}\right\}$ of models defined over $\mathbb{R}^{p}$ and taking their values in $\mathbb{R}^{q}$, the high-fidelity model $f^{(K+1)}=f^{\star}$ being the last element of the set. The hierarchy of model is such that for $k=1, \ldots, K$, we have $d_{k}<d_{k+1} \in \mathcal{K}$, with $d_{K+1}=q$ and $\mathcal{K}$ denoting a prescribed set of ordered positive integers lower or equal to $q$.

From the computational standpoint, in the case $f^{\star}(x)$ is a finite element discretization of an elleptic PDE with $r$ affine dependences in the bilinear form parametrized by 
$x$, computing a Petrov-Galerkin reduced-basis approximation $f^{(k)}$ at point $x$ requires off-line $\mathcal{O}\left(r d_{k} q^{2}\right)$ operations and, more importantly, it requires on-line $\mathcal{O}\left(d_{k}^{2}\left(r+d_{k}\right)\right)$ operations. Computing the high-fidelity solution requires instead $\mathcal{O}\left(q^{3}\right)$ operations, which suggests that an important computation gain for RB approximation if $d_{k} \ll q$. We remark that the complexity is slightly higher for a least-square reduced-basis approximation. The possibility to devise an off-line/on-line decomposition relies on the assumption of affine parametric dependence. Nevertheless, similar low on-line complexities are achievable in the case of non-affine parametric dependence using approximations by means of the empirical interpolation method. We refer to [15] for details and further extensions to the case of non-linear PDEs with non-affine parametric dependences.

Finally, we assume a score function $\phi: \mathbb{R}^{p} \rightarrow \mathbb{R}$ of the form $\phi(x)=h\left(f^{\star}(x)\right)$, where $h: \mathbb{R}^{q} \rightarrow \mathbb{R}$. Using the reduced models, we define score function approximations $\phi^{(k)}: \mathbb{R}^{p} \rightarrow \mathbb{R}$ as $\phi^{(k)}(x)=h\left(f^{(k)}(x)\right)$. Therefore, the hierarchy $\mathcal{F}$ is related to a sequence of approximations $\mathcal{M}=\left\{\phi^{(1)}, \phi^{(2)}, \ldots, \phi^{(K)}, \phi^{(K+1)}\right\}$, where the $(K+1)$-th element is the original score function $\phi^{(K+1)}=\phi$.

2.3. Third Ingredient: Error Bounds. For any $x \in \mathcal{X}$, we further assume that we can compute on-line for $k=1, \ldots, K$ an error bound of the form

$$
\left\|f^{\star}(x)-f^{(k)}(x)\right\|_{2} \leq \epsilon_{k}(x),
$$

with a complexity independent of the high-fidelity model dimension. Although the evaluation of error bounds can be in some cases expensive, error estimators for RB approximation involves generally low-complexity computations and are largely employed to steer adaptive schemes, see e.g., [15] and references therein. Indeed, taking advantage of the previously introduced off-line/on-line decomposition, it is typically possible to compute efficiently for any $x \in \mathcal{X}$ a local error bound for each subspace approximation. A posteriori error estimates [15] are computable on-line with a quadratic complexity in $d_{k}$ and $r$. More precisely, the error norm of the RB approximation is bounded by the ratio of the dual norm of the residual and the stability factor. The dual norm of the residual can typically be evaluated on-line with $\mathcal{O}\left(r^{2} d_{k}^{2}\right)$ operations, exploiting affine [15, Chapter 3.7] or approximate affine [15, Chapter 10.4] parametric dependance. On the other hand, provided an off-line characterization by interpolatory radial basis functions, the stability factor term is evaluated on-line with a complexity linear in the number of interpolation points and independent of $q$.

Now, in the case $h$ is a bounded linear function, by definition of an operator norm, we have $\left|\phi(x)-\phi^{(k)}(x)\right| \leq\|h\|_{2,2}\left\|f^{\star}(x)-f^{(k)}(x)\right\|_{2}$. In the case $h$ is the sup norm, using the triangular inequality and the fact that $f^{\star}(x) \in \mathbb{R}^{q}$ with $q$ finite, we obtain $\left|\phi(x)-\phi^{(k)}(x)\right| \leq\left\|f^{\star}(x)-f^{(k)}(x)\right\|_{\infty} \leq\left\|f^{\star}(x)-f^{(k)}(x)\right\|_{2}$. Using the error estimate 2.10 , we can then bound the error on any subset $\mathcal{Z}$ of $\mathcal{X}$ :

$$
\forall x \in \mathcal{Z}, \quad\left|\phi(x)-\phi^{(k)}(x)\right| \leq \alpha_{k}(\mathcal{Z})=c \max _{x^{\prime} \in \mathcal{Z}} \epsilon_{k}\left(x^{\prime}\right) .
$$

where $c=\|h\|_{2,2}$ or $c=1$ respectively for linear operators or for the sup norm. For ease of notations, when dealing with a distribution $\nu \in \mathcal{V}$, we will use the simplified notation $\alpha_{k}(\nu)$, instead of $\alpha_{k}(\operatorname{supp}(\nu))$.

We mention that the goal-oriented adaptive strategy proposed in 14 refines the error bound estimate (2.11). The idea of such a strategy is to directly rely on a posteriori estimates of the score function approximation error in order to infer a sequence of subspaces yielding fine approximations of the score function close to the set of rare events of interest and coarse approximations far away from it. 

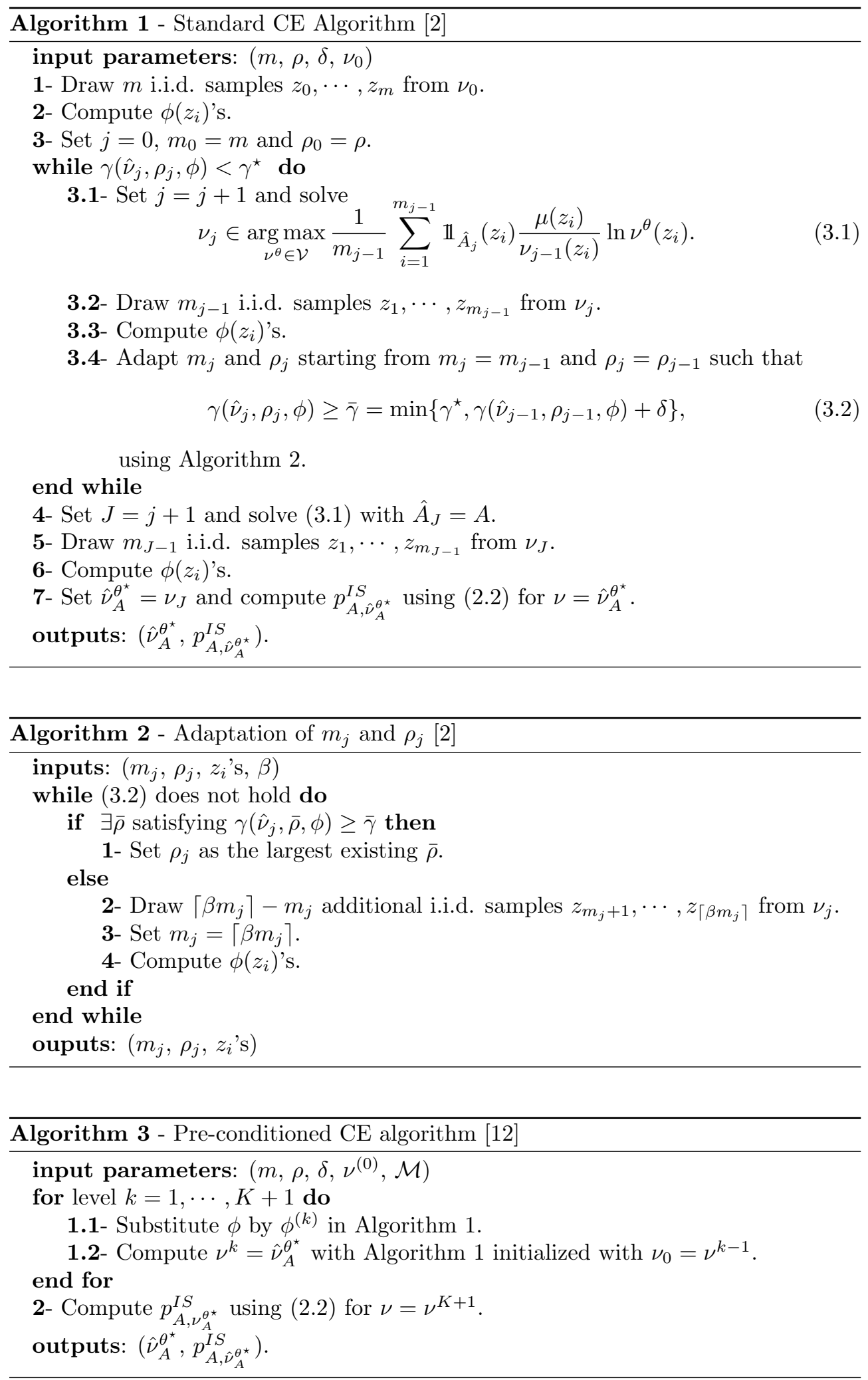
3. State-of-the-Art Recipes. Incorporating one after the other the two first ingredients in IS, we obtain the standard and the pre-conditioned CE algorithms. Note that we review hereafter algorithms which are certified to converge to the optimal biasing distribution. In consequence, we do not detail the hybrid method suggested in [13], although it relies on the same ingredients and is related, up to some extent.

3.1. Standard CE Algorithm. The standard CE method is exposed in Algorithm 1. The algorithm builds a sequence of proposal distributions starting from an initial distribution $\nu_{0}$ and ending with the optimal proposal $\hat{\nu}_{A}^{\theta^{\star}}$.

We detail hereafter this construction. In the first steps of the algorithm, $m$ samples are drawn from the initial proposal $\nu_{0}$ (in step 1) and related scores are computed (in step 2). Then, the algorithm estimates (in step 3) a sequence of proposals $\left\{\nu_{1}, \ldots, \nu_{J-1}\right\}$, and the related sequences of quantile parameters $\left\{\rho_{1}, \ldots, \rho_{J-1}\right\}$ and sample sizes $\left\{m_{1}, \ldots, m_{J-1}\right\}$, until the quantile reaches the desired score $\gamma^{\star}$. More precisely, at the $j$-th iteration, given $\rho_{j} \in(0,1)$, a sample size $m_{j}$ and a proposal $\nu_{j}$, the algorithm builds the empirical $\left(1-\rho_{j}\right)$-quantile

$$
\gamma\left(\hat{\nu}_{j}, \rho_{j}, \phi\right)=\max \left\{s \in \mathbb{R}:\left(\frac{1}{m_{j}} \sum_{i=1}^{m_{j}} \mathbb{1}_{\phi\left(z_{i}\right)<s}\right) \leq 1-\rho_{j}\right\},
$$

where $z_{1}, \cdots, z_{m_{j}}$ are i.i.d. samples from $\nu_{j}$. This quantile defines the stochastic event

$$
\hat{A}_{j+1}=\left\{x \in \mathbb{R}^{p}: \phi(x) \geq \min \left(\gamma\left(\hat{\nu}_{j}, \rho_{j}, \phi\right), \gamma^{\star}\right)\right\} .
$$

Given the set $\hat{A}_{j+1}$ and the proposal $\nu_{j}$, a refined distribution $\nu_{j+1}$ is estimated (in step 3.1) by solving (3.1), which is a MC approximation of the cross-entropy minimization problem (2.9). Then, updated samples are drawn according to the new proposal $\nu_{j+1}$ (in step 3.2) and related scores are computed (in step 3.3). The next quantile parameter $\rho_{j+1}$ and sample size $m_{j+1}$ are tuned (in step 3.4) in order to satisfy an increase of at least $\delta>0$ of the quantile. After $J-1$ iterations, the quantile $\gamma\left(\hat{\nu}_{J-1}, \rho_{J-1}, \phi\right)$ is above the desired score $\gamma^{\star}$. The optimal proposal $\hat{\nu}_{A}^{\theta^{\star}}=\nu_{J}$ can then be computed (in step 4) using the original set $A$. Samples are drawn (in step 5) according to $\hat{\nu}_{A}^{\theta^{*}}$ and related scores are computed (in step 6). The rare event probability is finally estimated by IS using these samples and scores (in step 7).

The free parameters of the algorithm are the initial sample size $m=m_{0}$, the initial quantile parameter $\rho=\rho_{0} \in(0,1)$, the minimal quantile increase value $\delta$ and the initial proposal $\nu_{0}$, which is in most cases set to the distribution of $X\left(i . e ., \nu_{0}=\mu\right)$.

Let us make some important comments. The algorithm imposes through condition (3.2) that quantiles will strictly increase at each of the algorithm iterations, implying that the estimated sequences of $\nu_{j}$ 's, $\rho_{j}$ 's and $m_{j}$ 's will be associated to a finite set of stochastic events satisfying, as the sets in (2.8), a nesting property $\hat{A}_{1} \supseteq \hat{A}_{2} \supseteq \cdots \supseteq A$. This condition is used to prove the convergence of the algorithm as shown in Section 5 .

To guarantee the validity of 3.2 at each iteration, we use an upgraded version of the $\mathrm{CE}$ algorithm first proposed in [2, Algorithm 5.2]. The difference with the basic CE method [4, Algorithm 2.1] is that the algorithm adapts at the $j$-th iteration the parameter $\rho_{j}$ and the sample size $m_{j}$ defining the sequence of nested events (3.4). This adaptation is presented in Algorithm 2. It consists in checking if $\rho_{j}$ can be tuned (in step 1) to meet the condition (3.2). Otherwise the sample size $m_{j}$ is increased (in step 3) by factor say $\beta=1.25$, i.e., new samples are drawn (in step 2) and new scores are computed (in step 4) until the condition is satisfied. The need for this adaptation step for convergence is justified by the asymptotic analysis exposed in Section 5 
Concerning complexity, the evaluation of the $\phi\left(z_{i}\right)$ 's in Algorithm 1 or in Algorithm 2 requires $\mathcal{O}\left(m q^{3}\right)$ operations. It represents in general the most computational demanding step of the $\mathrm{CE}$ algorithm. In the favorable situation where the problem (3.1) boils down to solving a linear system (e.g., for $\mathcal{V}$ being the family of $p$-dimensional Gaussians, see Section 6.3, an update of the proposal density requires $\mathcal{O}\left(p^{3}\right)$ operations, yielding for $p \ll q$ the overall complexity $\mathcal{O}\left(J m q^{3}\right)$.

3.2. Pre-conditioned CE Algorithm. In the multi-fidelity method proposed in 12 and detailed in Algorithm 3 , the biasing densities are refined solving a sequence of "low-fidelity CE methods". For $k=1, \ldots, K+1$, the distribution $\nu^{k}$ is obtained (in step 1.2) solving the optimization problem related to a CE method defined (in step 1.1) with score function approximation $\phi^{(k)} \in \mathcal{M}$ instead of $\phi$, with $\mathcal{M}$ gathering in principle any type of surrogates. The strategy proposed by the authors is to use the distribution obtained as the solution of $k$-th optimization problem of this sequence as an initialization for the problem at level $k+1$. After running sequentially the $K$ "low-fidelity CE methods", the optimal proposal $\hat{\nu}_{A}^{\theta^{*}}$ is obtained by running the standard CE method initialized with the $K$-th optimization problem's solution $\nu^{K}$. The rare event probability is finally estimated by IS (in step 2).

We remark that at each of the $K$ iterations of Algorithm 3, the pre-conditioned method invokes Algorithm 1, which resorts itself to the adaptation step performed by Algorithm 2. We notice that if one of the $K$ score function approximations is upper bounded by a value lower than $\gamma^{\star}$, a straightforward implementation of Algorithm 3 will yield a non convergent estimator. Indeed, in such a situation, there will exist an index $j^{\prime}$ such that for any $j \geq j^{\prime}$ the condition $\gamma\left(\hat{\nu}_{j}, \rho_{j}, \phi^{(k)}\right) \geq$ $\min \left\{\gamma^{\star}, \gamma\left(\hat{\nu}_{j-1}, \rho_{j-1}, \phi^{(k)}\right)+\delta\right\}$ will never hold even for an infinite sample size. To avoid this pathological case, the adaptation of the sample size is avoided in the $K$ first iterations of Algorithm 3 and replaced by an increment of $k$, i.e., a refinement from $\phi^{(k)}$ to $\phi^{(k+1)}$.

The main advantage of the pre-conditioned CE algorithm is that, using this sound initialization, Algorithm 1 (where $\phi^{(k)}$ substitutes $\phi$ ) typically converges at level $k$ in only a few iterations. As shown in [12] and mentioned in the following, some guarantees can be obtained under mild conditions on the number of iterations saved at each of the $K+1$ levels of Algorithm 3. Since this saving occurs in particular at the last level (i.e., at $K+1$ ), it partially alleviates the computational bottleneck induced by calling too many times the high-fidelity model. The algorithm complexity remains of $\mathcal{O}\left(J m q^{3}\right)$, as for the standard CE method. However, to obtain the initialization of Algorithm 3 at the last level, $K$ optimization problems need first to be solved, each one of them targeting a solution of the form (2.6) where $\phi^{(k)}$ substitutes $\phi$. In fact, the proposed pre-conditioning does not necessarily guarantee that the global computational cost is lower or equal to that of the standard CE method.

4. Recipe for Selecting Reduced Models in the CE Method. We propose in the following to incorporate the third ingredient and select the score approximation in a hierarchy of pre-computed surrogates, at each level of the CE optimization process.

4.1. The Proposed Algorithm. We present the proposed CE method in Algorithm 4. It relies on a relaxed set of events defined for $j \geq 0$ as

$$
\hat{A}_{j+1}^{\left(k_{j}\right)}=\left\{x \in \mathbb{R}^{p}: \phi^{\left(k_{j}\right)}(x) \geq \min \left(\gamma\left(\hat{\nu}_{j}, \rho_{j}, \phi^{\left(k_{j}\right)}\right)-2 \alpha_{k_{j}}\left(\hat{\nu}_{j}\right), \gamma^{\star}+\alpha_{k_{j}}\left(\hat{\nu}_{j}\right)\right)\right\},
$$

with the error bounds 


$$
\alpha_{k}\left(\hat{\nu}_{j}\right)=c \max _{i=1, \cdots, m_{j}} \epsilon_{k_{j}}\left(z_{i}\right)
$$

where $c$ is the constant in (2.11) and $z_{1}, \cdots, z_{m_{j}}$ are i.i.d. samples from $\nu_{j}{ }^{2}$. As described below, the algorithm determines the sequences of $k_{j}$ 's, $m_{j}$ 's, $\rho_{j}$ 's and $\nu_{j}$ 's. The idea behind Algorithm 4 is to compute, as for the standard CE method, a sequence of $\left(\nu_{j}, \rho_{j}, m_{j}\right)$ 's satisfying (3.2) or equivalently a sequence of nested events $\hat{A}_{j+1}$ including the target set of rare events $A$. This computation should rely as much as possible on the score function approximations and be controlled by their error estimates. In other words, the philosophy is to exploit the approximations and error bounds to adapt sequences of $k_{j}$ 's, $m_{j}$ 's, $\rho_{j}$ 's and $\nu_{j}$ 's so that $\hat{A}_{j+1}^{\left(k_{j}\right)}$ is the smallest set built with the score approximation $\phi^{\left(k_{j}\right)}$ which contains $\hat{A}_{j+1}$ : for any $z \in \mathbb{R}$, the set $\left\{x \in \mathbb{R}^{p}: \phi^{\left(k_{j}\right)}(x) \geq z, x \in \hat{A}_{j+1}\right\}$ should contain or be equal to $\hat{A}_{j+1}^{\left(k_{j}\right)}$.

4.1.1. General structure. Algorithm 4 is pretty much similar to Algorithm 1 , Its 7-steps structure differs slightly from the standard CE method, by the use of selected score approximations. We hereafter comment each step of the algorithm. At the beginning, $m$ samples are drawn (in step 1) from the initial proposal $\nu_{0}$ and related score approximations and error bounds are computed (in step 2). Then, the algorithm estimates (in step 3) a sequence of proposals $\left\{\nu_{1}, \ldots, \nu_{J-1}\right\}$, and related sequences of quantile parameters $\left\{\rho_{1}, \ldots, \rho_{J-1}\right\}$, sample sizes $\left\{m_{1}, \ldots, m_{J-1}\right\}$ and approximation levels $\left\{k_{1}, \ldots, k_{J-1}\right\}$, until the quantile computed with approximation $\phi^{\left(k_{j}\right)}$ reaches the desired score $\gamma^{\star}$ augmented by the error bound $\alpha_{k_{j}}\left(\hat{\nu}_{j}\right)$ (as proved in Section 5, the addition of this term guarantees that the quantile computed with the original $\phi$ is above $\left.\gamma^{\star}\right)$. Let us detail the building of these sequences. At the $j$-th iteration, given $\rho_{j} \in(0,1)$, a sample size $m_{j}$, a proposal $\nu_{j}$ and an approximation level $k_{j}$, the algorithm evaluates the empirical $\left(1-\rho_{j}\right)$-quantile $\gamma\left(\hat{\nu}_{j}, \rho_{j}, \phi^{\left(k_{j}\right)}\right)$ using samples $z_{1}, \cdots, z_{m_{j}}$ from $\nu_{j}$. This quantile together with the related error bound define the stochastic event $\hat{A}_{j+1}^{\left(k_{j}\right)}$ through 4.1. Given the set $\hat{A}_{j+1}^{\left(k_{j}\right)}$ and the proposal $\nu_{j}$, a refined distribution $\nu_{j+1}$ is estimated (in step 3.1) by solving 4.3), which is a MC approximation of the cross-entropy minimization problem 2.9 using the $\phi^{\left(k_{j}\right)}$ in place of the original score function $\phi$. Then, updated samples are drawn according to the new proposal $\nu_{j+1}$ (in step 3.2) and related score approximations and error bounds are computed (in step 3.3). The next quantile parameter $\rho_{j+1}$ and sample size $m_{j+1}$ are tuned (in step 3.4), together with the approximation level $k_{j+1}$, in order to satisfy a quantile increase of at least $\delta+2 \alpha_{k_{j}}\left(\hat{\nu}_{j}\right)$ (as shown in Section 5 , the addition of the term $2 \alpha_{k_{j}}\left(\hat{\nu}_{j}\right)$ guarantees that the quantile computed with the original $\phi$ increases of $\delta)$. After $J-1$ iterations, the quantile $\gamma\left(\hat{\nu}_{J-1}, \rho_{J-1}, \phi^{\left(k_{J-1}\right)}\right)$ is above the score $\gamma^{\star}+\alpha_{k_{J-1}}\left(\hat{\nu}_{J-1}\right)$. The end of the algorithm is identical to the standard CE method: the optimal proposal $\hat{\nu}_{A}^{\theta^{\star}}=\nu_{J}$ is computed (in step 4) using the original set $A$; samples are drawn (in step 5) according to $\hat{\nu}_{A}^{\theta^{*}}$ and related scores are computed (in step 6); the rare event probability is finally estimated by IS using these samples and scores (in step 7).

The free parameters of the algorithm are the same as for the standard CE method, supplemented by the hierarchy $\mathcal{M}$ of score approximations.

\footnotetext{
${ }^{2}$ We will see in Appendix $\mathrm{C}$ that $\alpha_{k}\left(\hat{\nu}_{j}\right)$ converges as $m_{j} \rightarrow \infty$ to $\alpha_{k_{j}}\left(\operatorname{supp}\left(\nu_{j}\right)\right)$, i.e., to a global error estimator for a distribution with infinite support. However, in practice, as $m_{j}$ is finite, $\alpha_{k}\left(\hat{\nu}_{j}\right)$ is rather a local error bound computed with samples drawn in the region where distribution $\nu_{j}$ has a lot of mass.
} 

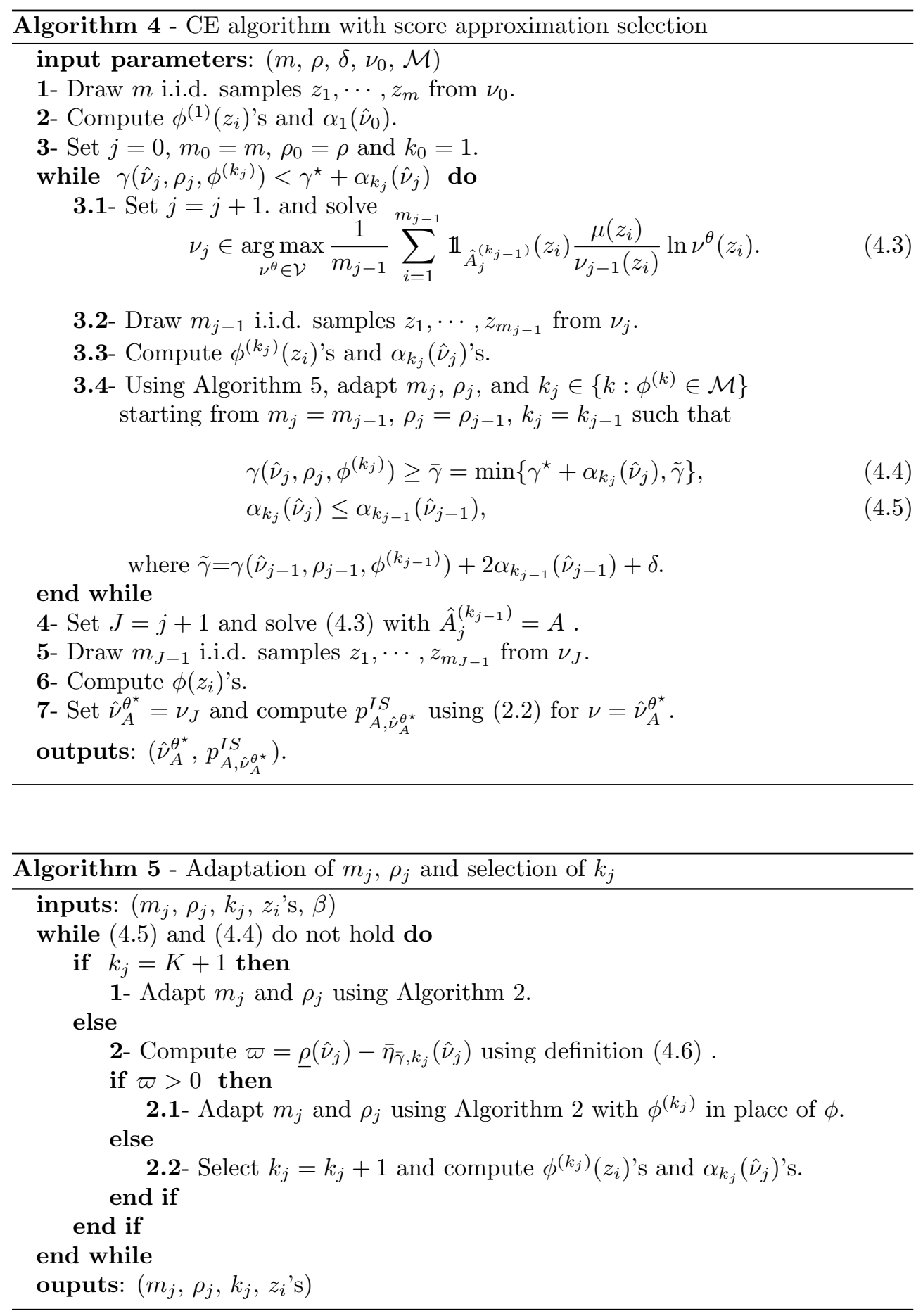

Finally, let us point out that problem 4.3 may be ill-conditioned, i.e., that the solution $\nu_{j}$ might not be unique. The non-uniqueness might be in certain circumstances only the consequence of an insufficient sample size, the circumstances being determined by the parametric distribution family $\mathcal{V}$. This issue is discussed in the 
case of the $p$-dimensional Gaussian family in Section 6.4

4.1.2. Selection of Score Approximations. The adaptation step is driven by Algorithm 5 , in place of Algorithm 2 in the standard CE method, and includes the selection of the score approximation. In the case $k_{j}=K+1$, i.e., no score approximation are used, Algorithm 5 boils down to Algorithm 2 (step 1). In other situations, the algorithm checks if the current approximation $\phi^{\left(k_{j}\right)}$ allows for the existence of a non-zero $\rho_{j}$ satisfying the nesting property and agreeing with an error tolerance, taking the form of conditions (4.4) and (4.5). As shown in Section 5.4.1 these two conditions guarantee asymptotically an increase of the quantile computed with the original high-fidelity score of at least $\delta$.

However, it is important to remark that at the current approximation level $k_{j}$, the condition $\gamma\left(\hat{\nu}_{j}, \rho_{j}, \phi^{\left(k_{j}\right)}\right) \geq \bar{\gamma}$ may in some cases only be satisfied for $\rho_{j}=0$, even if we increase the sample size $m_{j}$. This situation occurs in particular in the case where $\phi^{\left(k_{j}\right)}$ under-estimates systematically the score function. However, we do not know a priori if we are in such an undesirable situation. Rather than increasing $m_{j}$ and trying to obtain in vain an inaccessible non-zero value $\rho_{j}$, it is more cautious to use the current sample size and select the next possible approximation level in $\mathcal{M}$, for which there exists $\rho>0$ such that the condition $\gamma\left(\hat{\nu}_{j}, \rho, \phi^{\left(k_{j}\right)}\right) \geq \bar{\gamma}$ stands.

Ideally, in order to make the selection of $k_{j}$ more robust, we should check the existence of $\rho>0$ such that $\gamma\left(\nu_{j}, \rho, \phi^{\left(k_{j}\right)}\right) \geq \bar{\gamma}$ holds, and not only rely on the samples of the empirical distribution $\hat{\nu}_{j}$. We derive hereafter a worst-case sufficient condition for the existence of such an ideal condition, worst-case meaning that the approximated score under-estimates systematically the high-fidelity score of a value equal to the error bound. The worst-case sufficient condition is derived from the assumption that $\rho_{\bar{\gamma}}=\left\langle\mathbb{1}_{\phi(\cdot) \geq \bar{\gamma}}, \nu_{j}\right\rangle>0$ (which is a mild assumption used in Section 5.1 to prove convergence). Indeed as shown in Appendix A, a sufficient condition in this worstcase scenario is that $\rho_{\bar{\gamma}}>\eta_{\bar{\gamma}, k_{j}}$, where $\eta_{\bar{\gamma}, k_{j}}$ is the probability that the approximated score falls into the interval $\left[\gamma\left(\nu_{j}, \rho_{\bar{\gamma}}, \phi^{\left(k_{j}\right)}\right), \gamma\left(\nu_{j}, \rho_{\bar{\gamma}}, \phi^{\left(k_{j}\right)}\right)+\alpha_{k_{j}}\left(\nu_{j}\right)\right]$.

In practice, $\rho_{\bar{\gamma}}$ and $\eta_{\bar{\gamma}, k_{j}}$ are both inaccessible as they depend on the high-fidelity score $\phi$ on one side, and on the other side on intractable integrals. To overcome this issue, we first derive a lower bound on $\rho_{\bar{\gamma}}-\eta_{\bar{\gamma}, k_{j}}$ using the following remark, and design a tractable plug-in estimator of this bound.

REMARK 1. Let $\alpha_{k}(\nu)$ and $\alpha_{\ell}(\nu)$ be error bounds such that $\alpha_{k}(\nu) \leq \alpha_{\ell}(\nu)$. From $\left|\phi^{(k)}(x)-\phi(x)\right| \leq \alpha_{\ell}(\nu)$, we deduce $\left\langle\mathbb{1}_{\phi(\cdot)<s-\alpha_{\ell}(\nu)}, \nu\right\rangle \leq\left\langle\mathbb{1}_{\phi^{(k)}(\cdot)<s}, \nu\right\rangle \leq\left\langle\mathbb{1}_{\phi(\cdot)<s+\alpha_{\ell}(\nu)}, \nu\right\rangle$, which in turns leads to $\left|\gamma\left(\nu, \rho, \phi^{(k)}\right)-\gamma(\nu, \rho, \phi)\right| \leq \alpha_{\ell}(\nu)$ for any $\rho \in(0,1)$.

Therefore, a lower bound for $\rho_{\bar{\gamma}}$ and an upper bound for $\eta_{\bar{\gamma}, k_{j}}$ are available, from which we design the related plug-in estimators, respectively defined as

$$
\begin{aligned}
\underline{\rho}_{\bar{\gamma}}\left(\hat{\nu}_{j}\right) & =\left\langle\mathbb{1}_{\phi^{\left(k_{j}\right)}(\cdot) \geq \bar{\gamma}+\alpha_{k_{j}}\left(\hat{\nu}_{j}\right)}, \hat{\nu}_{j}\right\rangle, \\
\bar{\eta}_{\bar{\gamma}, k_{j}}\left(\hat{\nu}_{j}\right) & =\max _{\gamma^{\prime} \in\left[\gamma_{b}, \gamma_{u}\right]}\left\langle\mathbb{1}_{\phi^{\left(k_{j}\right)}(\cdot) \in\left[\gamma^{\prime}, \gamma^{\prime}+\alpha_{k_{j}}\left(\hat{\nu}_{j}\right)\right]}, \hat{\nu}_{j}\right\rangle,
\end{aligned}
$$

with $\gamma_{b}=\gamma\left(\hat{\nu}_{j}, \bar{\rho}_{\bar{\gamma}}\left(\hat{\nu}_{j}\right), \phi^{\left(k_{j}\right)}\right), \gamma_{u}=\gamma\left(\hat{\nu}_{j}, \underline{\rho}_{\bar{\gamma}}\left(\hat{\nu}_{j}\right), \phi^{\left(k_{j}\right)}\right)$ and $\bar{\rho}_{\bar{\gamma}}\left(\hat{\nu}_{j}\right)=\left\langle\mathbb{1}_{\phi^{\left(k_{j}\right)}(\cdot) \geq \bar{\gamma}-\alpha_{k_{j}}\left(\hat{\nu}_{j}\right)}, \hat{\nu}_{j}\right\rangle$. We observe that the positivity of $\varpi=\underline{\rho}_{\bar{\gamma}}\left(\hat{\nu}_{j}\right)-\bar{\eta}_{\bar{\gamma}, k_{j}}\left(\hat{\nu}_{j}\right)$ implies in particular the validity of condition 4.4, making the first condition more demanding than the second. The criterion for the selection of the approximation level is thus more exigent than the quantile increase condition used once an approximation level has been selected. 
Therefore, computing $\varpi$ (in step 2) and checking its positivity constitutes our criterion for the selection of the next approximation level $k_{j}$. More precisely, if $\varpi>0$ and the level $k_{j}$ is selected, the algorithm adapts (in step 2.1) $\rho_{j}$ and $m_{j}$ such that $\gamma\left(\hat{\nu}_{j}, \rho_{j}, \phi^{\left(k_{j}\right)}\right) \geq \bar{\gamma}$ using Algorithm 2 with $\phi^{\left(k_{j}\right)}$ in place of $\phi$. In the case where $\varpi \leq 0, k_{j}$ is increased (in step 2.2) to the next possible value in $\mathcal{K}$, i.e., a refined score function approximation $\phi^{\left(k_{j}\right)}$ is selected in $\mathcal{M}$. This refinement process continues until the condition $\varpi>0$ is met or the highest level $k_{j}=K+1$ (for which $\phi^{(K+1)}=\phi$ ) is reached.

4.2. Computational Saving. In comparison to state-of-the-art, the main innovation of Algorithm 4 is that it selects at each of its iteration the score function approximation in a hierarchy of pre-computed surrogates to meet the need for current accuracy. More precisely, the proposed algorithm uses score function approximations directly within the core of the CE method, and furthermore parsimoniously guided by error estimates (in particular all score approximations are not systematically used).

As for the standard $\mathrm{CE}$ algorithm, the computation of the high-fidelity score function or of its approximations represents the most computational intensive steps. Of course, the evaluation of the high-fidelity score function for a set of samples is irrevocable in the final step for the estimation of the rare event probability. Nevertheless, in the previous iterations of the proposed algorithm, surrogates are chosen sparingly, in order to significantly reduce the computational burden. As shown in the analysis of Section 5, the computational gain is not at the expense of a deterioration of the sequence of proposal densities. Indeed, the sequence of $\nu_{j}$ 's generated by the proposed algorithm are asymptotically guaranteed to satisfy the quantile increase condition (3.2) and converge to the optimal density (2.6), just as for the standard CE algorithm.

The algorithm complexity remains of $\mathcal{O}\left(J m q^{3}\right)$, as for state-of-the-art methods. In theory, we note that if the bound on the maximum number of iterations of Algorithm 4 and Algorithm 1 are identical (and we will see that this is the case in the next section), then the worst computational load involved by the former is guaranteed to be lower or equal to the one involved by the latter. In practice, we expect a large number of iterations to be performed with the reduced models, i.e., requiring only $\mathcal{O}\left(m d_{k}^{3}\right)$ operations, in the usual setting where $r \leq d_{k}$ with $r$ the number of affine parameters.

Let us point out that the error bounds are computed in $\mathcal{O}\left(m r^{2} d_{k}^{2}\right)$ operations. Therefore, this does not increase significantly the computational cost as long as $r^{2} \ll d_{k}$. This regime is in general the only one suitable for importance sampling, which becomes very challenging in high dimensions as pointed out in [16. Besides, we remark that the evaluation of $\bar{\eta}_{\bar{\gamma}, k_{j}}\left(\hat{\nu}_{j}\right)$ at the core of the the selection of the approximation level involves a simple 1-dimensional minimization. Once the score function approximations have been computed, it can be solved with a negligible computational load using standard optimization techniques.

Finally, we remark that making an on-line adaptation of the directions (in addition of the dimension) of the $\mathrm{RB}$ spaces is prohibitive if we want to maintain an on-line cubic complexity in $d_{k}$, independent of the ambient dimension $q$. Indeed, updating a direction in the reduced model would require $\mathcal{O}\left(r d_{k} q^{2}\right)$ operations, see Section 2.2. Nevertheless, the algorithm's efficiency could be increased reconsidering the off-line/on-line decomposition paradigm, designing so-called adaptive reduced models built by enrichment of the RB spaces as proposed in 8, 14. This possible enhancement of the algorithm is however out of the scope of the present work. 
5. Asymptotic Analysis. We provide theoretical guarantees on the convergence of the proposed algorithm. We begin by introducing assumptions and reviewing state-of-the-art results.

5.1. Assumptions. We use the following assumptions, as done in [2].

Assumption A: For any $\nu^{\theta} \in \mathcal{V}, \operatorname{supp}(\mu) \subseteq \operatorname{supp}\left(\nu^{\theta}\right)$.

In words, Assumption A ensures that the domination relation $\operatorname{supp}(\mu) \subseteq \operatorname{supp}\left(\nu_{A}^{\theta^{*}}\right)$, satisfied by the zero-variance density $\nu_{A}^{\theta^{\star}}$ given by $(2.6)$ is also satisfied for any feasible $\nu^{\theta} \in \mathcal{V}$. This assumption is trivially satisfied if densities in $\mathcal{V}$ have an infinite support.

We remark that Assumption A implies that $\left\langle\mathbb{1}_{A}, \nu^{\theta}\right\rangle>0$ for any $\nu^{\theta} \in \mathcal{V}$ as long as $\left\langle\mathbb{1}_{A}, \mu\right\rangle>0$, i.e., as long as the sought rare-event probability is non-zero. We mention that this assumption could be relaxed as done in [2].

Assumption B: We assume that

i) the set $\Theta$ is compact;

ii) for almost every $\theta$, the function $\ln \nu^{\theta}$ is continuous on $\Theta$;

iii) there exists a function $h: \mathbb{R}^{p} \rightarrow \mathbb{R}$ such that $\langle h, \mu\rangle<\infty$ and $\left|\ln \nu^{\theta}(x)\right| \leq h(x)$ for all $x \in \mathbb{R}^{p}$ and $\nu^{\theta} \in \mathcal{V}$;

The properties $i$ ), ii) and iii) are satisfied by numerous family of distributions. To illustrate that, consider the Gaussian family $\mathcal{V}=\left\{\nu^{\theta}: \nu^{\theta}(x)=1 / \theta e^{\left(-\|x\|_{2}^{2} / \theta\right)}, \theta \in \Theta \subset\right.$ $\mathbb{R}^{+}$, where $\Theta$ is a compact excluding zero. In this case, we verify that Assumption B holds, and in particular there exists a quadratic function $h$ satisfying iii).

5.2. Previous Results. We hereafter expose the convergence result for the standard CE method [2].

Theorem 5.1 (Homem de Mello and Rubinstein, 2002). Suppose that Assumptions $A$ and $B$ hold. Then, in Algorithm 1 as $m_{j} \rightarrow \infty$,

- the biasing distribution $\hat{\nu}_{A}^{\theta^{*}}$ converges almost surely to (2.6) in a number of iterations lower or equal to

$$
\hat{J}_{\max }=\left\lceil\frac{\gamma^{\star}-\gamma\left(\hat{\nu}_{0}, \rho_{0}, \phi\right)}{\delta}\right\rceil+1 ;
$$

- the $S C V$ of the estimator $(2.2)$ is

- at the $j$-th iteration using $\nu=\nu_{j}$ and assuming $\nu_{\hat{A}_{j}}^{\star} \in \mathcal{V}$

$$
\frac{1}{p_{A}^{2}} \mathbb{E}\left[\left(p_{A, \nu_{j}}^{I S}-p_{A}\right)^{2}\right]=\frac{1}{m_{j} p_{A}} p_{\hat{A}_{j} \backslash A} ;
$$

- equal to zero at the $J$-th iteration using $\nu=\nu_{J}$ and assuming $\nu_{A}^{\star} \in \mathcal{V}$.

The almost sure convergence in at most $\hat{J}_{\max }$ iterations is proven in 2]. The result on the estimator SCV after the $j$-th iteration is straightforward, as detailed in Appendix $\mathrm{D}$ and obvious after the $J$-th iteration as the algorithm sets $\hat{A}_{j}=A$ implying that $p_{\hat{A}_{j} \backslash A}=0$. We see that, the smaller the set $\hat{A}_{j} \backslash A$, the smaller the variance. Therefore, it is tempting to build a sequence $\hat{A}_{j}$ tending quickly to $A$ which would yield a drop-off on the estimator variance. However, the drawback of such a 
construction is that this approach can be expensive since it generally requires large values for the $m_{j}$ 's. Indeed, the value of $m_{j}$ may need in this situation to be increased significantly until it fulfils condition (3.2). In fact, there is a tradeoff between the value of $m_{j}$ 's and parameter $\delta$ which imposes a minimal speed of convergence of the sequence of $\hat{A}_{j}$ 's towards $A$.

We mention that the authors in [12, Proposition 1] slightly enhance the first statement of Theorem 5.1. They prove that, under mild conditions, the bound on the maximum number of iterations becomes lower or equal to $\hat{J}_{\max }$ when their multi-fidelity approach is used to initialize the $\mathrm{CE}$ method. The assumption guaranteeing that the bound on the maximum number of iterations is lowered at level $k$ (and in particular at the last level $K+1$ using the high-fidelity model) is that $\left\langle\mathbb{1}_{\phi^{(k)}(\cdot) \geq \gamma\left(\nu^{k-1}, \rho, \phi^{(k)}\right)}, \nu^{k-1}\right\rangle \geq\left\langle\mathbb{1}_{\phi^{(k)}(\cdot) \geq \gamma\left(\nu^{k-1}, \rho, \phi^{(k)}\right)}, \mu\right\rangle$. In practice, this assumption holds if we can verify conditions on the score function approximation regularity and on its cumulative distribution with respect to the biasing densities in $\mathcal{V}$, see [12, Assumption 1 - 2]. Nevertheless, there exists no theoretical guarantees that the $K+1$ level of the pre-conditioned method are more efficient than the standard CE method.

5.3. Our Result. We now present the theoretical guarantees obtained with the proposed method in the asymptotic regime.

Theorem 5.2. Suppose that Assumptions $A$ and B hold. Then, in Algorithm 4 as $m_{j} \rightarrow \infty$,

- the biasing distribution $\hat{\nu}_{A}^{\theta^{*}}$ converges almost surely to (2.6) in at most $\hat{J}_{\max }$ iterations, as for the standard CE method;

- the $S C V$ of the estimator 2.2 is

- at the $j$-th iteration using $\nu=\nu_{j}$ and assuming $\nu_{\hat{A}_{j}^{\left(k_{j-1}\right)}}^{\star} \in \mathcal{V}$

$$
\frac{1}{p_{A}^{2}} \mathbb{E}\left[\left(p_{A, \nu_{j}}^{I S}-p_{A}\right)^{2}\right]=\frac{1}{m_{j} p_{A}}\left(p_{\hat{A}_{j} \backslash A}+p_{\hat{A}_{j}^{\left(k_{j-1}\right)} \backslash \hat{A}_{j}}\right),
$$

which is the minimal SCV achievable among all feasible $\hat{A}_{j}^{\left(k_{j-1}\right)}$ 's;

- equal to zero at the $J$-th iteration using $\nu=\nu_{J}$ and assuming $\nu_{A}^{\star} \in \mathcal{V}$, as for the standard CE method.

In the theorem, we call the tuple $\left(\nu_{j}, \rho_{j}, k_{j}\right)$, or equivalently the set $\hat{A}_{j+1}^{\left(k_{j}\right)}$ given in (4.1) feasible if and only if $\left(\nu_{j}, \rho_{j}\right)$ satisfies (3.2). The first statement of this theorem proves the almost sure convergence of our algorithm towards the optimal biasing distribution in at most $\hat{J}_{\max }$ iterations, as the standard CE algorithm. In this worst case scenario of $\hat{J}_{\max }$ iterations, we deduce that Algorithm 4 will use the same or lower computation power than the standard CE method, since at each iteration the score function can be exchanged for an approximation of lower complexity.

The second statement of this theorem shows that the squared coefficient of variations of the estimator 2.2 obtained with the biasing distribution at the $j$-th iteration of Algorithm 1 and Algorithm 4 are identical up to an additional term proportional to the probability of the set $\hat{A}_{j}^{\left(k_{j-1}\right)} \backslash \hat{A}_{j}$. Furthermore, the statement claims that this probability is the minimal achievable among all feasible $\hat{A}_{j}^{\left(k_{j-1}\right)}$. In other words, given the score functions approximation $\phi^{\left(k_{j-1}\right)}$ and the worst-case error $\alpha_{k}\left(\hat{\nu}_{j}\right)$, the choice of the set $\hat{A}_{j}^{\left(k_{j-1}\right)}$ is optimal at the $j$-th iteration in the sense that no other feasible set can yield a lower variance of the IS estimator. 
According to this second statement, the use of a relaxed set containing $\hat{A}_{j}$ (even if chosen optimally in the feasible set) instead of $\hat{A}_{j}$ itself (as done in the standard $\mathrm{CE}$ algorithm) implies unavoidably an additional term in the SCV. However, it is important to point out that the choice of the relaxed set at the $j$-th iteration of the algorithm should not necessarily be guided by obtaining the lowest achievable SCV (as done in the standard CE algorithm). The criterion to be minimized is rather the global computational cost for the estimation of the target: the optimal biasing density $\nu_{J}$. Indeed, whatever the chosen path for the $\nu_{j}$ 's in the distribution space from $\nu_{0}=\mu$ to $\nu_{J}$ and whatever their associated SCV's, $\nu_{J}$ will always be associated with the same SCV, independently of this path and the involved algorithm. Therefore, the objective is to obtain the optimal density with a minimum of effort. In this perspective, the proposed algorithm builds at the $j$-th iteration the relaxed set $\hat{A}_{i}^{\left(k_{j-1}\right)} \supseteq \hat{A}_{j}$ for inference of $\nu_{j}$ so that 1) the standard quantile increase condition (3.2) holds, 2) the estimation involves the score function approximation of lowest complexity. Thanks to these two properties, the optimal density (2.6) is reached by the proposed algorithm in at most $\hat{J}_{\max }$ iterations, just as for the standard CE algorithm, but with possibly a minimal computation burden.

\subsection{Proof of Theorem 5.2 ,}

5.4.1. First Statement: Convergence. Proof. This statement is a straightforward consequence of the two propositions given in this section.

Proposition 5.3. Under assumptions $A$ and $B$, Algorithm 5 selects $\left\{\rho_{j}\right\}_{j=1}^{J}$, $\left\{k_{j}\right\}_{j=1}^{J}$ and $\left\{m_{j}\right\}_{j=1}^{J}$ such that the sequence $\left\{\left(\nu_{j}, \rho_{j}\right)\right\}_{j=1}^{J}$ produced by Algorithm 4 satisfies almost surely as $m_{j} \rightarrow \infty$ the condition

$$
\gamma\left(\nu_{j}, \rho_{j}, \phi\right) \geq \min \left\{\gamma^{\star}, \gamma\left(\nu_{j-1}, \rho_{j-1}, \phi\right)+\delta\right\} .
$$

Proof. We begin by verifying that Algorithm 5 searches at each iteration the smallest $k_{j}$ such that 4.5 holds and $\varpi>0$. Note that the latter condition holds only if $\rho\left(\hat{\nu}_{j}\right)>0$, which in turns implies the existence of $\rho_{j}>0$ such that (4.4). The proof of Proposition 5.3 then relies on the two following arguments. On the one hand, statements i) and ii) in Lemma C.1 show that, for $m_{j}$ large enough, conditions 4.4 and 4.5 imply their deterministic counterparts

$$
\begin{aligned}
& \gamma\left(\nu_{j}, \rho_{j}, \phi^{\left(k_{j}\right)}\right) \geq \min \left\{\gamma^{\star}+\alpha_{k_{j}}\left(\nu_{j}\right), \tilde{\gamma}_{\ell}\right\}, \\
& \alpha_{k_{j}}\left(\nu_{j}\right) \leq \alpha_{\ell}\left(\nu_{j-1}\right),
\end{aligned}
$$

where $\tilde{\gamma}_{\ell}=\gamma\left(\nu_{j-1}, \rho_{j-1}, \phi^{(\ell)}\right)+2 \alpha_{\ell}\left(\nu_{j-1}\right)+\delta$. On the other hand, using Remark 1 to major $\gamma\left(\nu_{j}, \rho_{j}, \phi^{\left(k_{j}\right)}\right)$ and minor $\gamma\left(\nu_{j-1}, \rho_{j-1}, \phi^{(\ell)}\right)$, it is straightforward to verify that conditions (5.3) and (5.4) imply that $\left(\nu_{j}, \rho_{j}\right)$ satisfies (5.2).

In words, this first proposition shows that the sequence $\left\{\left(\nu_{j}, \rho_{j}\right)\right\}_{j=0}^{J}$ built by the proposed algorithm satisfy asymptotically the deterministic counterpart of the quantile increase condition 3.2 used in the standard CE method. In particular, it shows that events $A_{1} \supset A_{2} \supset \cdots \supset A_{J}=A$, are nested according to 5.2 where $J<J_{\max }$.

Proposition 5.4. If the sequence $\left\{\left(\nu_{j}, \rho_{j}\right)\right\}_{j=1}^{J}$ satisfies 5.2 then under Assumption $B$, the biasing distribution $\hat{\nu}_{A}^{*}$ given by Algorithm 4 converges to the optimal one (2.6) almost surely as $m_{j} \rightarrow \infty$ in $J \leq \hat{J}_{\max }$ iterations. 
Proof. To show this result we introduce the deterministic counterpart of problem $(4.3)$, that is

$$
\underset{\nu^{\theta} \in \mathcal{V}}{\arg \max }\left\langle\mathbb{1}_{A_{j}^{\left(k_{j-1}\right)}} \ln \nu^{\theta}, \mu\right\rangle
$$

where $A_{j}^{\left(k_{j-1}\right)}=$

$\left\{x \in \mathbb{R}^{p}: \phi^{\left(k_{j-1}\right)}(x) \geq \min \left(\gamma\left(\nu_{j-1}, \rho_{j-1}, \phi^{\left(k_{j-1}\right)}\right)-2 \alpha_{k_{j-1}}\left(\nu_{j-1}\right), \gamma^{\star}+\alpha_{k_{j-1}}\left(\nu_{j-1}\right)\right)\right\}$.

We show in Appendix $\mathrm{C}$ that under Assumption B, the distribution $\nu_{j}$ in $\mathrm{Al}$ gorithm 4 (the solution (4.3) ) converges almost surely as $m_{j} \rightarrow \infty$ to the solution (5.5). This result corresponds to statement iii) of Lemma C.1. Moreover, because the sequence $\left\{\left(\nu_{j}, \rho_{j}\right)\right\}_{j=1}^{J}$ satisfies 5.2 according to Proposition 5.3, we have $\gamma\left(\nu_{j-1}, \rho_{j-1}, \phi\right) \leq \gamma\left(\nu_{j}, \rho_{j}, \phi\right)+\delta$ for $j=1, \cdots, J$. And at the $(J-1)$-th iteration we have $\gamma\left(\nu_{J-1}, \rho_{J-1}, \phi\right) \geq \gamma^{\star}$. Thus, step 4 of Algorithm 4 provides the optimal distribution $\arg \max _{\nu^{\theta} \in \mathcal{V}}\left\langle\mathbb{1}_{A} \ln \nu^{\theta}, \mu\right\rangle$. Finally, it is clear from (5.2) and the almost sure convergence of quantile and error bound approximations (statements $i$ ) and ii) of Lemma C.1 that for $m$ large enough, the number of iterations of the algorithm, i.e., $J=\min \left\{j \geq 1: \gamma\left(\hat{\nu}_{j}, \rho_{j}, \phi^{\left(k_{j}\right)}\right) \geq \gamma^{\star}+\alpha_{k_{j}}\left(\hat{\nu}_{j}\right)\right\}+1$, is upper bounded by $\hat{J}_{\max }$ defined in (5.1), i.e., $J \leq \hat{J}_{\max }$.

Relying on Remark 1 in Section 4.1.2, we can compute in practice an upper bound on this maximum number of iteration

$$
\hat{J}_{\max } \leq\left\lceil\frac{\gamma^{\star}-\gamma\left(\hat{\nu}_{0}, \rho_{0}, \phi^{\left(k_{0}\right)}\right)-\alpha_{k_{0}}\left(\hat{\nu}_{0}\right)}{\delta}\right\rceil+1,
$$

without resorting to the high-fidelity model and the original score function $\phi$.

5.4.2. Second Statement: Minimal Squared Coefficient of Variation. We present hereafter the proof of the second part of Theorem 5.2, i.e., which shows that, for a number of samples large enough, the squared coefficient of variation given by the theorem is the minimal achievable for all feasible $\hat{A}_{j}^{\left(k_{j-1}\right)}$ 's.

Proof. On the one hand, we show in Remark 2 of Appendix B that by construction we have the inclusion $A_{j}^{\left(k_{j-1}\right)} \supseteq A$. Moreover, from the almost sure convergence of quantile and error bound approximation (statements $i$ ) and ii) of Lemma C.1), we know that $\hat{A}_{j}^{\left(k_{j-1}\right)}$ converges almost surely to $A_{j}^{\left(k_{j-1}\right)}$ as $m \rightarrow \infty$. Therefore, we deduce that the inclusion $\hat{A}_{j}^{\left(k_{j-1}\right)} \supseteq A$ holds asymptotically. This inclusion is a necessary condition to obtain a finite variance since we can not guarantee that $A \backslash \hat{A}_{j}^{\left(k_{j-1}\right)} \nsubseteq$ $\operatorname{supp}(\mu)^{3}$. On the other hand, by following the reasoning of Appendix $\mathrm{D}$ where $p_{\hat{A}_{j}^{\left(k_{j-1}\right)}}$ substitutes for $p_{\hat{A}_{j}}$, we see that assuming $\nu_{\hat{A}_{j}^{\left(k_{j-1}\right)}} \in \mathcal{V}$ and $m_{j}$ large enough, the variance writes after the $j$-th iteration as $\mathbb{E}\left[\left(p_{A, \nu_{j}}^{I S}-p_{A}\right)^{2}\right]=\frac{p_{A}}{m_{j}}\left(p_{\hat{A}_{j}^{\left(k_{j-1}\right)}}-p_{A}\right)$. The variance obviously vanishes after the $J$-th iteration as the algorithm sets $\hat{A}_{j}^{\left(k_{j-1}\right)}=A$.

\footnotetext{
${ }^{3}$ Indeed, in the case we build a sequence of feasible $\hat{A}_{j}^{\left(k_{j-1}\right)}$ 's such that we have $\hat{A}_{j}^{\left(k_{j-1}\right)} \nsupseteq A$ and $A \backslash \hat{A}_{j}^{\left(k_{j-1}\right)} \subseteq \operatorname{supp}(\mu)$, using the assumption $\nu_{\hat{A}_{j}^{\left(k_{j-1}\right)}}^{{ }^{\prime}} \in \mathcal{V}$ we have the infinite variance $\mathbb{E}\left[\left(p_{A, \nu_{j}}^{I S}-p_{A}\right)^{2}\right]=\frac{1}{m_{j}}\left(\left\langle\mathbb{1}_{A} \frac{\mu}{\nu_{j}}, \mu\right\rangle-p_{A}^{2}\right)=\frac{1}{m_{j}}\left(p_{\hat{A}_{j}^{\left(k_{j-1}\right)}}\left\langle\frac{\mathbb{1}_{A}}{\mathbb{1}_{\hat{A}_{j}^{\left(k_{j-1}\right)}}}, \mu\right\rangle-p_{A}^{2}\right)$.
} 
Let us verify that this quantity is minimal among all feasible $\hat{A}_{j}^{\left(k_{j-1}\right)}$. In Remark 3 of Appendix B, we show that a necessary condition to obtain a finite variance is that $A_{j}^{\left(k_{j-1}\right)} \supseteq A_{j}$. Since $A_{j} \supseteq A$, the probability $p_{A_{j}^{\left(k_{j-1}\right)} \backslash A}$ is minimal if the probability $p_{A_{j}^{\left(k_{j-1}\right)} \backslash A_{j}}$ is the lowest achievable. For $m_{j}$ large enough, this is equivalent to search the minimal $p_{\hat{A}_{j}^{\left(k_{j-1}\right)} \backslash \hat{A}_{j}}$ among all feasible $\hat{A}_{j}^{\left(k_{j-1}\right)}$. The result follows since, by construction the set $\hat{A}_{j}^{\left(k_{j-1}\right)}$ is the smallest feasible set such that $\hat{A}_{j}^{\left(k_{j-1}\right)} \supseteq \hat{A}_{j}$, implying that the probability $p_{\hat{A}_{j}^{\left(k_{j-1}\right)} \backslash \hat{A}_{j}}$ is the smallest achievable.

6. Numerical Evaluation: Pollution Alerts. We consider a mass transfer problem describing the behavior of pollutant released by industrial chimneys into the atmosphere, or by a plant in a river. The concentration of the pollutant evolves in a compact domain $\Omega \subseteq \mathbb{R}^{2}$. We are interested in the rare event probability that the maximum value of the concentration of the pollutant exceeds a given value in the domain.

6.1. Physical Problem. As detailed in [15, Chapter 8.4], the evolution of the pollutant is modeled by an advection-diffusion-reaction equation, while the emission is described by a parametrized source term. The pollutant concentration function $f: \Omega \times \mathbb{R}^{p} \rightarrow \mathbb{R}^{+}$is ruled by the following PDE:

$$
\left\{\begin{aligned}
-\kappa_{1} \Delta f(z, x)+b(z, x) \cdot \nabla f(z, x)+a_{0} f(z, x) & =s(z, x), & & z \in \Omega \\
\kappa_{1} \nabla f(z, x) \cdot n(z) & =0, & & z \in \partial \Omega,
\end{aligned}\right.
$$

parametrized by the $p$-dimensional vector $x=\left(x_{1}^{\top}, x_{2}, x_{3}\right)^{\top}$ with $x_{1} \in \mathbb{R}^{p-2}$ and $x_{2}, x_{3} \in \mathbb{R}$. We considered the domain $\Omega=\left[\begin{array}{ll}0 & 1\end{array}\right] \times\left[\begin{array}{ll}0 & 1 / 2\end{array}\right]$. The normal to the domain boundary $\partial \Omega$ is denoted by vector $n(z), \kappa_{1}=0.03$ represents the molecular diffusivity of the chemical species and $a_{0}=0.5$ represents the intensity of reaction processes. Here, $b(z, x)$ is a turbulent motion field parametrized by $p-2$ coefficients gathered in vector $x_{1}$. More precisely, $b(z, x)$ is the (zero-mean) divergence-free fractional Brownian motion (fBm) proposed in [17, Proposition 3.1] of Hurst exponent $H=\frac{1}{3}$, supplied by an additional constant field, see Figure 6.1. The fBm admits an affine parametrization with $p-3$ real uncorrelated wavelet coefficients distributed according to the standard normal law, while the constant field is of unit magnitude and parametrized by a wind direction angle. The number of wavelet coefficients parameterizing the $\mathrm{fBm}$ is related to the number of elements considered in the truncated wavelet series, and in turns determines the motion field resolution. The source $s(z, x)=\exp \left(-\frac{\left(z_{2}-x_{2}\right)^{2}+\left(z_{3}-x_{3}\right)^{2}}{\kappa_{2}^{2}}\right)$, with $z=\left(z_{2}, z_{3}\right)^{\top} \in \Omega$ describes the pollutant emission, characterized in terms of its position $\left(x_{2}, x_{3}\right)^{\top}$ and its spreading $\kappa_{2}$. Parameter $x$ will be drawn according to a $p$-dimensional Gaussian random distribution $\mu$. The setup for the Gaussian parameters will be detailed in Section 6.4.

We consider the weak formulation of (6.1) and consider high-fidelity approximations $f^{\star}: \Omega \times \mathbb{R}^{p} \rightarrow \mathbb{R}^{+}$of its solutions via a finite-element method, see details in [15. Chapter 2.5].

We are interested in the rare event probability that the maximum value of the concentration of the pollutant over the domain exceeds a given value $\gamma^{\star}$. We thus define the probability $p_{A}$ with $A=\left\{x \in \mathbb{R}^{p}: \phi(x) \geq \gamma^{\star}\right\}$, where the score function is defined as the sup norm, i.e., $\phi(x)=\left\|f^{\star}(\cdot, x)\right\|_{\infty}$. 

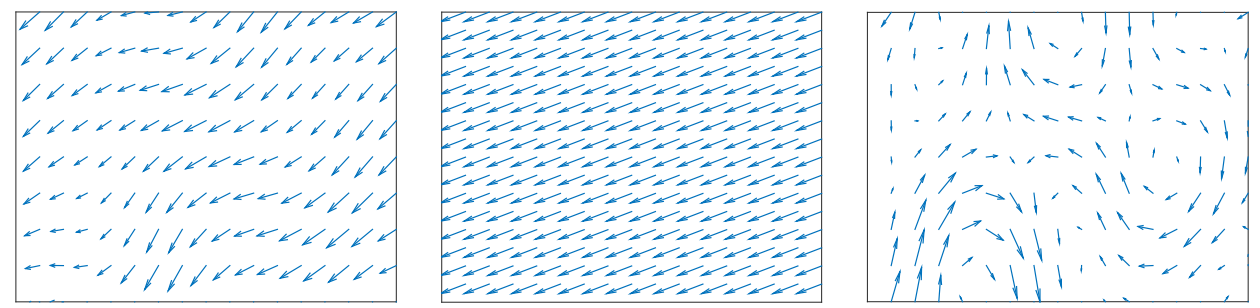

FIG. 6.1. Turbulent motion field. For a sample $x$, motion $b(z, x)$ (left) decomposed into a constant field (middle) and a divergence-free fBm (right).

6.2. Reduced Basis Approximations. We use principal orthogonal decomposition (POD) to compute the set of nested approximation sub-spaces $\left\{V_{k}\right\}_{k=1}^{K}$, where $d_{K}=250$. We use 2000 representative solutions, so-called snapshots. Since $s(z, x)$ is nonlinear in $x$, problem (6.1) does not fulfill the assumption of affine parametric dependence necessary to take advantage of the off-line/on-line decomposition. In consequence, we set up an approximate affine expansion by means of empirical interpolation method using 30 interpolation points. In this manner, we generate for any $x$ and any $k$ the $d_{k}$-dimensional RB approximation $f^{(k)}(z, x)$ of the high fidelity solution $f^{\star}(z, x)$, see details in [15, Chapter 10.5]. Examples of reduced basis approximations are displayed in Figure 6.2. We fix a hierarchy of reduced model $f^{(k)} \in \mathcal{F}$ related to the set of dimensions $\mathcal{K}$. The different choices for the set $\mathcal{K}$ will be detailed in Section 6.4. The a posteriori error estimate $\epsilon_{k}(x)$ at point $x$ for the reduced model $f^{(k)}$ is computed using an off-line/on-line decomposition [15].

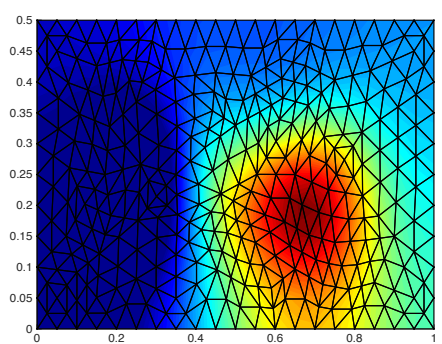

$$
d_{k}=5
$$

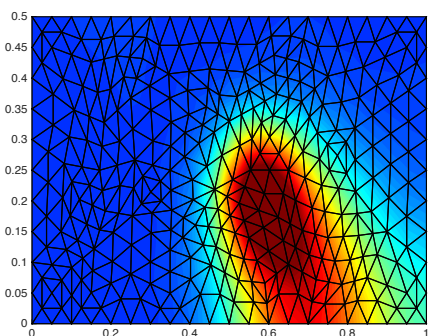

$$
d_{k}=50
$$

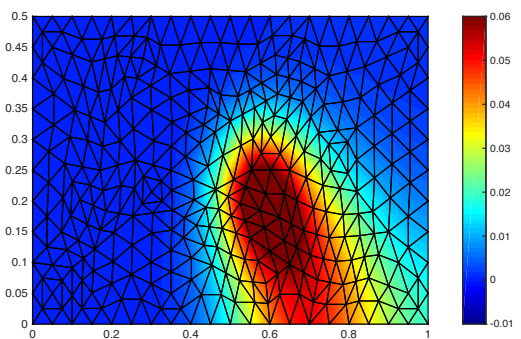

High-fidelity $(q=648)$

FIG. 6.2. Approximation of pollutant concentration. For a sample $x$, mesh and solution by Galerkin projections $f^{(k)}(z, x)$ or by the high-fidelity model $f(z, x)$ (with spreading $\kappa_{2}=0.15$ ).

Accordingly we derive for $k=1, \cdots, K$, the score function approximation from the $\mathrm{RB}$ approximation $f^{(k)}$ as $\phi^{(k)}(x)=\left\|f^{(k)}(\cdot, x)\right\|_{\infty}$. Using $(4.2)$, this leads to bound the approximation error norm as $\alpha_{k}(\hat{\nu})=\max _{i=1, \cdots, m} \epsilon_{k}\left(z_{i}\right)$, where $z_{1}, \cdots, z_{m}$ are i.i.d. samples from $\nu \in \mathcal{V}$.

6.3. Parametrization of the $\mathbf{C E}$ methods. The set $\mathcal{V}$ is chosen to be the family of $p$-dimensional normal distributions

$$
\nu(\theta)=\frac{1}{\sqrt{\left|2 \pi \Sigma_{\nu}\right|}} \exp \left(-\frac{1}{2}\left(x-m_{\nu}\right)^{\top} \Sigma_{\nu}^{-1}\left(x-m_{\nu}\right)\right)
$$

parametrized by vector $\theta \in \mathbb{R}^{p(p+1)}$, gathering the components of the mean $m_{\nu} \in \mathbb{R}^{p}$ and of the covariance matrix $\Sigma_{\nu} \in\left\{M \in \mathbb{R}^{p \times p}:|M| \geq 0, M^{\top}=M\right\}$. On the one hand, we notice in this example that, unfortunately, the zero-variance biasing distribution $\nu_{A}^{\star}$ defined in 2.4 does not belong to the Gaussian family $\mathcal{V}$. Therefore, 
we expect the optimal distribution $\nu_{A}^{\theta^{*}} \in \mathcal{V}$ to have a non-zero variance. On the other hand, this family presents the advantage to yield a closed-form solution $\nu_{j}$ to problem (4.3). Indeed, cancelling the gradient of the objective function of (4.3), we can show that the parameters $\left(m_{\nu_{j}}, \Sigma_{\nu_{j}}\right)$ of the Gaussian distribution $\nu_{j}$ are solution of linear systems. More precisely, we find that $m_{\nu_{j}}$ is the solution of

$$
\sum_{i=1}^{m_{j-1}} \mathbb{1}_{\hat{A}_{j}^{\left(k_{j-1}\right)}}\left(z_{i}\right) \frac{\mu\left(z_{i}\right)}{\nu_{j-1}\left(z_{i}\right)}\left(z_{i}-m_{\nu_{j}}\right)=0
$$

while $\Sigma_{\nu_{j}}$ solves

$$
\sum_{i=1}^{m_{j-1}} \mathbb{1}_{\hat{A}_{j}^{\left(k_{j-1}\right)}}\left(z_{i}\right) \frac{\mu\left(z_{i}\right)}{\nu_{j-1}\left(z_{i}\right)}\left(I_{p}-\left(z_{i}-m_{\nu_{j}}\right)\left(z_{i}-m_{\nu_{j}}\right)^{\top} \Sigma_{\nu_{j}}^{-1}\right)=0,
$$

where $I_{p}$ is the p-dimensional identity matrix. We verify that the latter solution is feasible by construction, i.e., symmetric and positive semi-definite. Nevertheless, these systems are full-rank (implying a unique solution) only if sufficient $z_{i}$ 's (distributed according to $\nu_{j}$ ) belong to the set $\hat{A}_{j}^{\left(k_{j-1}\right)}$. Hopefully, it is straightforward to show that this happens for a sample size $m_{j-1}$ large enough.

6.4. Experimental Setup. We detail in this section practical considerations concerning our numerical simulations.

Distribution $\mu$ is chosen to be an uncorrelated $p$-dimensional Gaussian of mean $\left(\begin{array}{llllll}0.8 & 0.15 & 17 \pi / 18 & 0 & \cdots & 0\end{array}\right)^{\top}$ and characterized by an identity covariance matrix. We design the following experimental benchmark.

\begin{tabular}{c|c|c|c|c|c} 
Experiment & $q$ & $p$ & $\kappa_{2}$ & $\gamma^{\star}$ & $p_{A}$ \\
\hline$\# 1$ & 648 & 3 & 0.25 & 1.76 & $1.3 \times 10^{-6}$ \\
$\# 2$ & 648 & 3 & 0.25 & 1.8 & $1.2 \times 10^{-10}$ \\
$\# 3$ & 17024 & 13 & 0.15 & 1.62 & $2.4 \times 10^{-5}$ \\
$\# 4$ & 17024 & 21 & 0.15 & 1.62 & $2.1 \times 10^{-5}$
\end{tabular}

The solution of the discretized system, the RB approximations and the a posteriori error bounds are computed via the Matlab ${ }^{\circledR}$ toolbox "redbKIT" available at http://redbkit.github.io/redbKIT. We accelerate substantially a posteriori error estimation by rewriting the procedure with matrix products optimized for Matlab.

The rare event probabilities are estimated using:

- the standard CE method (Algorithm 1) described in 2,

- the pre-conditioned CE method (Algorithm 3) introduced in [12,

- the proposed CE method (Algorithm 4).

For each of thes experiments, in order to obtain (an approximation of) $p_{A}$ displayed in the previous table, we first compute reference probabilities by averaging $p_{A, \nu}^{I S}$ over a set of 30 estimates for experiment \#1 and \#2 (resp. 10 estimates for experiment \#3 and \#4), where each of the rare event probability estimates was obtained using the standard CE and a number of samples of $m=10^{4}$. We consider a set of specific values of $m \in \mathcal{M}=\left\{10^{3}, 2 \times 10^{3}, 4 \times 10^{3}, 6 \times 10^{3}, 8 \times 10^{3}, 10^{4}\right\}$ and the set $\mathcal{F}$ of models of dimension in the set $\mathcal{K}$. In particular, for experiment \#1 and $\# 2$ we identify $\mathcal{K}$ either to $\mathcal{K}_{2}=\{150, q\}, \mathcal{K}_{6}=\{50,100,150,200,250, q\}$ or $\mathcal{K}_{21}=\{50,60, \ldots, 250, q\}$, while for experiment $\# 3$ and $\# 4$ we identify $\mathcal{K}$ either to $\mathcal{K}_{2}=\{100, q\}, \mathcal{K}_{5}=\{100,125,150,175, q\}$ or $\mathcal{K}_{8}=\{50,75,100,125,150,175,200, q\}$.

We finally provide some additional details on the parametrization of the CE methods. The initial quantile parameter is set to $\rho=0.2$ and the minimal step size is chosen 
to be $\delta=10^{-2}$. We avoid the general tendency of densities ratio in the IS estimate to go to infinity for large values of $p$ [16, by setting the eigenvalues of the covariance matrix solving (6.2) to a minimal value of $5 \cdot 10^{-5}$ for experiments $\# 1, \# 2$ and $\# 3$ and of $5.10^{-4}$ for experiment \#4. Note that a similar strategy is adopted in [12].

Besides, we mention that in the context of the proposed CE method, we substituted $\alpha_{k_{j-1}}\left(\hat{\nu}_{j-1}\right)$ by $\alpha_{k_{j}}\left(\hat{\nu}_{j}\right)$ in $\tilde{\gamma}$ appearing in 4.4 to avoid unreachable values of $\tilde{\gamma}$ with the reduced model $f^{\left(k_{j}\right)}$ in the case the error comited with $f^{\left(k_{j-1}\right)}$ is too large in the first levels of the hierarchy. In the worst-case scenario, this substitution will yield $2\left(\alpha_{k_{j-1}}\left(\hat{\nu}_{j-1}\right)-\alpha_{k_{j}}\left(\hat{\nu}_{j}\right)\right) / \delta$ supplementary iterations in the proposed CE method.

6.5. Results. The quality and efficiency of rare event estimation is evaluated according to the following criteria.

- Variance. The estimator SCV defined in (2.3) was approximated by the empirical average over 10 estimates of $\left(p_{A, \nu}^{I S}-p_{A}\right)^{2} / p_{A}^{2}$. Here $p_{A}$ denotes the reference probability computed previously and $p_{A, \nu}^{I S}$ denotes the current rare event probability estimate.

- Runtime. This measurement is obtained by averaging the algorithm's total runtime in seconds over of a set of 10 rare event estimation processes. We use the 4 cores of a $2,8 \mathrm{GHz}$ Intel Core i7 processor with 16 GB RAM running a Matlab ${ }^{\circledR}$ parallelized implementation.

- Number of iterations. The number of iterations at each level of the CE algorithms was averaged over a set of 10 rare event estimation processes.
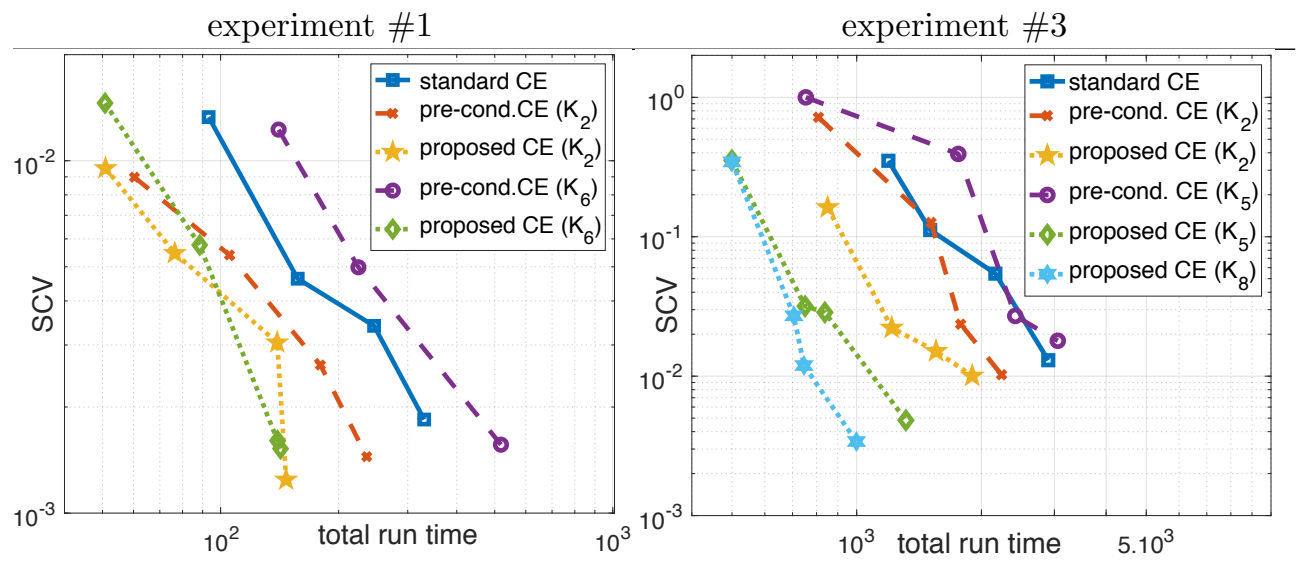

FIG. 6.3. Evaluation in terms of variance and runtime. Plots are obtained varying $m \in \mathcal{M}$ and $\mathcal{K} \in\left\{\mathcal{K}_{2}, \mathcal{K}_{6}\right\}$ (experiment \#1) or $\mathcal{K} \in\left\{\mathcal{K}_{2}, \mathcal{K}_{5}, \mathcal{K}_{8}\right\}$ (experiment \#3).

We show in Figure 6.3 the performance of the standard, pre-conditioned and proposed algorithms in terms of the estimator's SCV as a function of the overall algorithm runtime. Plots are obtained by varying the sample size $m \in \mathcal{M}$ and $\mathcal{K} \in$ $\left\{\mathcal{K}_{2}, \mathcal{K}_{6}\right\}$ for experiment $\# 1$ or $\mathcal{K} \in\left\{\mathcal{K}_{2}, \mathcal{K}_{5}, \mathcal{K}_{8}\right\}$ for experiment $\# 3$. The bar-plots of Figure 6.4 display the average number of iterations spent by the 3 different algorithms in each level of the reduced model hierarchies in the case $m=10^{4}$ for experiments \#1 and $\# 2, m=8.10^{3}$ for experiment $\# 3$ and $m=6.10^{3}$ for experiment $\# 4$.

We observe in Figure 6.3 that, in the case of experiment \#1, the use of a hierarchy of reduced models can lower significantly the algorithm runtime while yielding a similar SCV for the IS estimator. The magnitude of the gain reaches nearly half a decade, although the problem is characterized by a relatively moderate ratio between 
experiment \#1
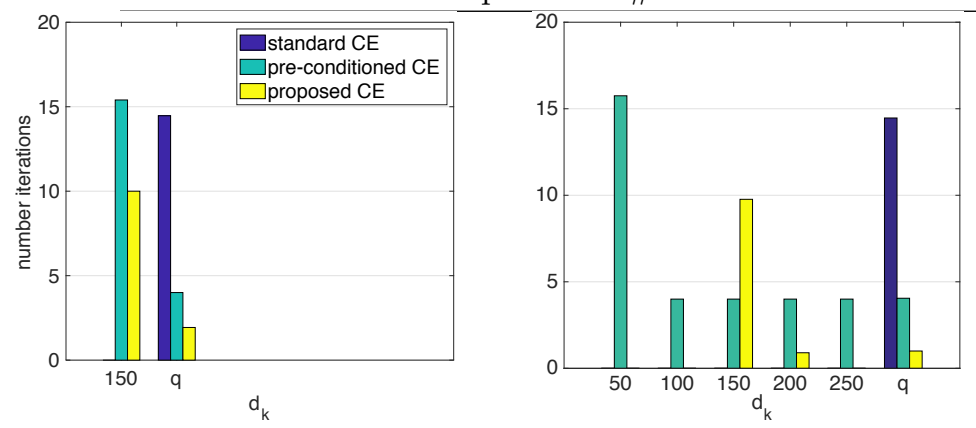

experiment \#3
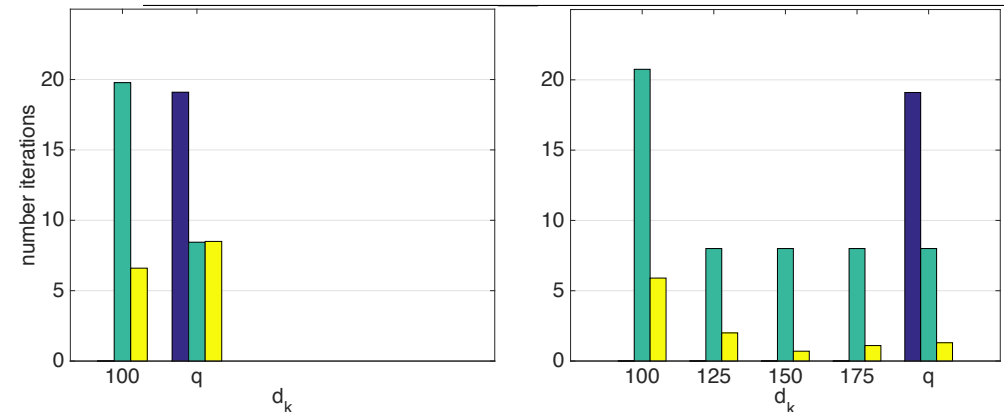

experiment \#2

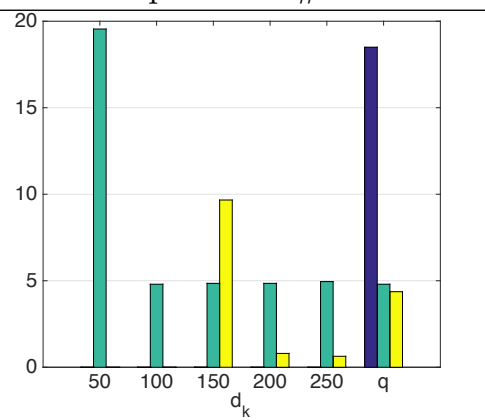

experiment \#4

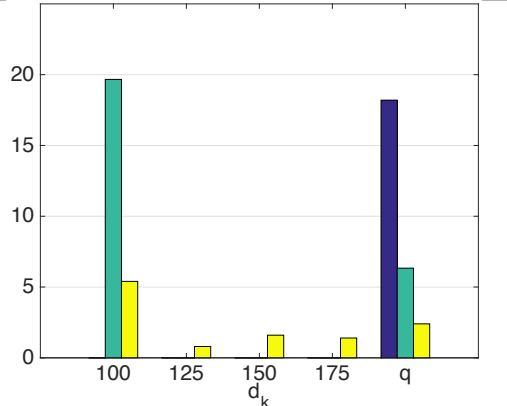

FIG. 6.4. Evaluation in terms of iteration number Average iteration number for the different algorithms at each level of the hierarchy of reduced models of dimension $d_{k}$ in the set $\mathcal{K}_{2}, \mathcal{K}_{5}$ or $\mathcal{K}_{6}$ (The most favorable hierarchy was chosen in experiment \#4, i.e., $\mathcal{K}_{2}$ and $\mathcal{K}_{5}$ respectively for the pre-conditioned and the proposed methods).

the dimension of the high-fidelity model $q=648$ and the dimensions of the reduced models. The pre-conditioned and the proposed methods both achieve similar performances in the case of a 2-level hierarchy. However, in the case of a 6-level hierarchy, although the proposed $\mathrm{CE}$ method still brings a significant gain, we remark that the pre-conditioned CE method fails in reducing (and even increases) the algorithm runtime. This illustrates the fact that selecting the reduced model accuracy in the CE method is mandatory to guarantee a runtime reduction in general. The bar-plots of Figure 6.4 show clearly that for $\mathcal{K}_{6}$, some levels of the hierarchy used by the preconditioned algorithm are ignored by our method, and furthermore, that our method tends to perform fewer iterations on the selected levels of the hierarchy. According to Figure 6.3, the accuracy of the two methods is similar. We conclude that, on the one hand, most of the levels used by the pre-conditioned method are useless and, on the other hand, it is unnecessary to iterate until convergence on each level of the hierarchy.

In experiment $\# 2$, we consider the lower probability in order of $10^{-10}$ (instead of $10^{-6}$ for experiment $\# 1$ ). Interestingly, in order to characterize this very rare event, the bar-plots of Figure 6.4 indicate that the proposed method with $\mathcal{K}=\mathcal{K}_{6}$ performs identically as for experiment \#1 on the first levels of the hierarchy (for $d_{k}=50,100,150$ and 200), and adds only a few iteration on the last levels (for $d_{k}=250$ and 648$)$. This behavior contrasts with the pre-conditioned method which spoils several iterations at each level of the hierarchy to reach convergence. Moreover, we mention that, for the proposed method, the run time acceleration is in order of $\times 2$ for experiment \#1 and \#2 (the SCV preserves the same order of magnitude). This is not the case for the pre-conditioned method, which slows down the estimation by a 
factor of about $2 / 3$ for experiment \#1, while an acceleration of about $3 / 2$ is observed for experiment \#2.

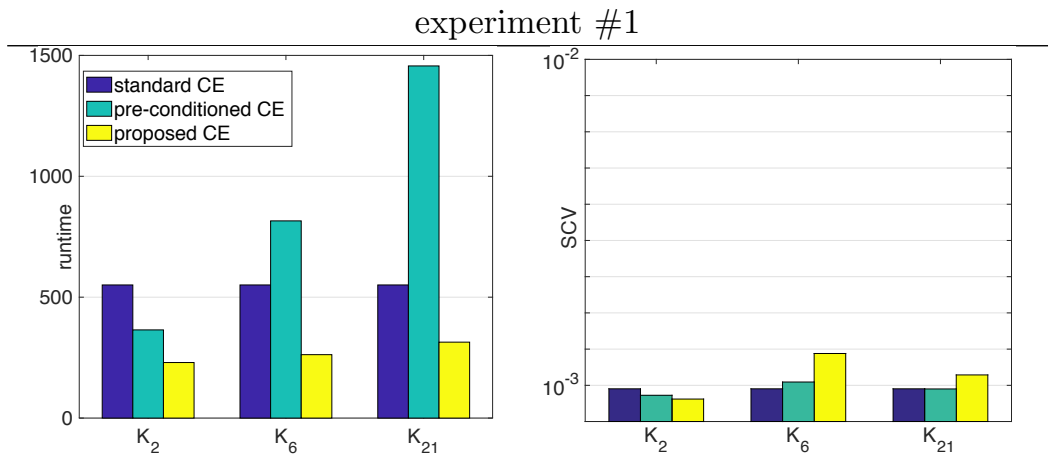

FIG. 6.5. Influence of the number of levels of the hierarchy. Results obtained with the different algorithms for a 2-, 6- or 21-level hierarchy denoted respectively $\mathcal{K}_{2}, \mathcal{K}_{6}$ or $\mathcal{K}_{21}$ for $m=10^{4}$.

To push our analysis further, we investigate the influence of the number of levels of the hierarchy on runtime and SCV. The bar-plots of Figure 6.5 show the influence related to experiments $\# 1$ for a sample size of $m=10^{4}$ with $\mathcal{K} \in\left\{\mathcal{K}_{2}, \mathcal{K}_{6}, \mathcal{K}_{21}\right\}$. We remark that the runtime of our method is stable as the number of levels in the hierarchy increase. The behavior of the pre-conditioned method is exactly at the opposite: runtime increases significantly with the number of levels. Nevertheless, this runtime stability or increase does not impact the quality of the estimation in experiments \#1 as shown by the stable behavior of the SCV.

Plots of Figure 6.3 related to experiment \#3, show the behavior of the algorithms when we increase by more than a factor 10 the dimensionality of both, the random field parameter and the simulation. More precisely, we substitute in model (6.1) a constant advection field $(p=3)$ for a divergence-free $\mathrm{fBm}(p=13)$ and we increase the number of finite elements from $q=648$ to $q=17024$. We still observe in this high-dimensional setting the good performance of the proposed method. The plot indicates that a hierarchy with at least 5 levels accelerates the rare event estimation process by a factor 3 . Using only 2 levels in the hierarchy slightly reduces the gain brought by the proposed method. The comparable performances obtained for a 5 - or 8-level hierarchy confirm the weak sensitivity of the method to the number of levels. In contrast, the pre-conditioned $\mathrm{CE}$ algorithm fails to accelerate the estimation process. In the best scenario, a 2-level hierarchy only succeeds to reach the performance of the standard CE method. As attested by the bar-plots in Figure 6.4 these poor results are induced by the waste of iterations used to reach convergence at each level of the hierarchy, which in turns drives away the biasing density from the optimal one. On the contrary, the proposed method tends to distribute and spare the iterations among the different levels of the hierarchy.

As shown in Figure 6.3 the behavior of the three different algorithms remains similar for experiment \#4 in terms of the number of iterations. However, the performances deteriorate significantly in terms of variance: the SCV of the proposed or the standard methods increase by a factor 4 while the one related to the pre-conditioned method explodes by a factor 50. This deterioration occurring when the dimension of the parameter space is increased up to $p=21$ is likely to be the effect of the curse of dimensionality prevailing for IS [16]. Nevertheless, the SCV of the proposed method remains about 4 time smaller than the SCV of the standard method, suggesting that our method is likely to be less sensitive to high-dimension settings. 
7. Conclusion. In this paper we have developed a computational strategy aiming to accelerate the IS estimation of a rare event probability, optimized with the $\mathrm{CE}$ method. At each level of the CE optimization process, a significant acceleration is obtained by selecting a surrogate from a hierarchy of score function approximations according to the need for accuracy. This need is quantified using certified bounds on the approximation error. An asymptotic analysis proves that the proposed algorithm is guaranteed to converge in a worst-case scenario at least as fast as state-of-the-art methods and that the reduced model selection is optimal in the sense it yields the minimal achievable estimator variance. In agreement with these theoretical arguments, numerical simulations quantify the gain brought by the proposed algorithm on a challenging pollutant transfer problem, where the high-fidelity score function is modeled by a PDE and surrogates using RB.

\section{Appendix A. A Sufficient Condition for Quantile Increase.}

Lemma A.1. If $\rho_{\bar{\gamma}}>\eta_{\bar{\gamma}, k_{j}}$, where $\rho_{\bar{\gamma}}=\left\langle\mathbb{1}_{\phi(\cdot) \geq \bar{\gamma}}, \nu_{j}\right\rangle$ and $\eta_{\bar{\gamma}, k_{j}}$ is the probability $\eta_{\bar{\gamma}, k_{j}}=\left\langle\mathbb{1}_{\left[\gamma\left(\nu_{j}, \rho_{\bar{\gamma}}, \phi^{\left(k_{j}\right)}\right), \gamma\left(\nu_{j}, \rho_{\bar{\gamma}}, \phi^{\left(k_{j}\right)}\right)+\alpha_{k_{j}}\left(\nu_{j}\right)\right]}, \nu_{j}\right\rangle$, then there exists $\rho>0$ such that $\gamma\left(\nu_{j}, \rho, \phi^{\left(k_{j}\right)}\right) \geq \bar{\gamma}$ holds.

Proof. if $\eta_{\bar{\gamma}, k_{j}}<\rho_{\bar{\gamma}}$, there exists $\rho \in\left(0, \rho_{\bar{\gamma}}-\eta_{\bar{\gamma}, k_{j}}\right]$ such that

$$
\begin{aligned}
\gamma\left(\nu_{j}, \rho, \phi^{\left(k_{j}\right)}\right) & =\max \left\{s \in \mathbb{R}:\left\langle\mathbb{1}_{\phi^{\left(k_{j}\right)}(\cdot)<s}, \nu_{j}\right\rangle \leq 1-\rho\right\}, \\
& \geq \max \left\{s \in \mathbb{R}:\left\langle\mathbb{1}_{\phi^{\left(k_{j}\right)}(\cdot)<s}, \nu_{j}\right\rangle \leq 1-\left(\rho_{\bar{\gamma}}-\eta_{\bar{\gamma}, k_{j}}\right)\right\}, \\
& =\max \left\{s \in \mathbb{R}:\left\langle\mathbb{1}_{\phi^{\left(k_{j}\right)}(\cdot)<s-\alpha_{k_{j}}\left(\nu_{j}\right)}, \nu_{j}\right\rangle \leq 1-\rho_{\bar{\gamma}}\right\}, \\
& \geq \max \left\{s \in \mathbb{R}:\left\langle\mathbb{1}_{\phi(\cdot)<s}, \nu_{j}\right\rangle \leq 1-\rho_{\bar{\gamma}}\right\}=\bar{\gamma} .
\end{aligned}
$$

The first equality is the quantile definition, the second equality follows from the definition of $\eta_{\bar{\gamma}, k_{j}}$ noticing that $\gamma\left(\nu_{j}, \rho_{\bar{\gamma}}, \phi^{\left(k_{j}\right)}\right)+\alpha_{k_{j}}\left(\nu_{j}\right)=\max \left\{s \in \mathbb{R}:\left\langle\mathbb{1}_{\phi^{\left(k_{j}\right)}(\cdot)<s-\alpha_{k_{j}}}, \nu_{j}\right\rangle \leq\right.$ $\left.1-\rho_{\bar{\gamma}}\right\}$, while the last one is obtained by making the change of variable $s^{\prime}=s-\alpha_{k_{j}}$ in the definition of the quantile and using the definition of $\rho_{\bar{\gamma}}$. The first inequality is due to the fact that the expectation $\left\langle\mathbb{1}_{\phi^{\left(k_{j}\right)}(\cdot)<s}, \nu_{j}\right\rangle$ is a non-decreasing function of $s$, while the last one is deduced from Remark 1 by bounding the worst-case error.

Appendix B. Inclusion Relations for the Relaxed Sets. We address two useful remarks. First, we notice that the sequence of relaxed sets includes the sequence of original sets.

REMARK 2. The definition of the sets implies the inclusion:

$$
A_{j} \subseteq A_{j}^{\left(k_{j-1}\right)} .
$$

Indeed, Remark 1 implies that $\gamma\left(\nu_{j-1}, \rho_{j-1}, \phi\right) \geq \gamma\left(\nu_{j-1}, \rho_{j-1}, \phi^{\left(k_{j-1}\right)}\right)-\alpha_{k_{j-1}}\left(\nu_{j-1}\right)$. If $x \in A_{j}$, then we obtain that

$\phi^{(k)}(x)+\alpha_{k_{j-1}}\left(\nu_{j-1}\right) \geq \phi(x) \geq \gamma\left(\nu_{j-1}, \rho_{j-1}, \phi\right) \geq \gamma\left(\nu_{j-1}, \rho_{j-1}, \phi^{\left(k_{j-1}\right)}\right)-\alpha_{k_{j-1}}\left(\nu_{j-1}\right)$,

which yields that $\phi^{\left(k_{j-1}\right)}(x) \geq \gamma\left(\nu_{j-1}, \rho_{j-1}, \phi^{\left(k_{j-1}\right)}\right)-2 \alpha_{k_{j-1}}\left(\nu_{j-1}\right)$, showing that $x \in A_{j}^{\left(k_{j}\right)}$ and therefore proving inclusion B.1. 
We then note that each element of the sequence of relaxed sets includes the target $A$.

Remark 3. By construction, we have $A \subseteq A_{j}$. Thus, condition B.1 guarantees that $A \subseteq A_{j}^{\left(k_{j-1}\right)}$. This condition is not only sufficient but also necessary to guarantee uniformly (over the class of certified reduced-models) that $A \subseteq A_{j}^{\left(k_{j-1}\right)}$, the latter condition being as already mentioned mandatory to obtain a finite variance of the IS estimator. Indeed, if condition (B.1) does not hold, we can always build a reduced model with a maximum error $\alpha_{k_{j}}$ such that $A \nsubseteq A_{j}^{\left(k_{j-1}\right)}$ although $A \subseteq A_{j}$.

\section{Appendix C. Consistency of MC approximations.}

Lemma C.1. As $m \rightarrow \infty$, almost surely

i) $\gamma\left(\hat{\nu}_{j}, \rho_{j}, \phi^{\left(k_{j}\right)}\right)$ converges to $\gamma\left(\nu_{j}, \rho_{j}, \phi^{\left(k_{j}\right)}\right)$,

ii) $\alpha_{k_{j}}\left(\hat{\nu}_{j}\right)$ converges to $\alpha_{k_{j}}\left(\nu_{j}\right)$,

iii) under Assumption B, 4.3. converges to (5.5).

Proof. Statement $i$ ) is shown in [2]. Statement ii) follows from Borel Cantelli Lemma [18. Indeed, denoting $M_{m_{j}}=\max \left(\epsilon_{k_{j}}\left(z_{1}\right), \ldots, \epsilon_{k_{j}}\left(z_{m_{j}}\right)\right)$ where $z_{i}$ 's are i.i.d. samples of $\nu_{j}$ and denoting $a=\max _{z \in \operatorname{supp}\left(\nu_{j}\right)} \epsilon_{k_{j}}(z)$, what needs to be shown is that $\lim _{m_{j} \rightarrow \infty} M_{m_{j}}=a$, with probability one. This happens to be the equivalent of a zero probability of the event $\left|M_{m_{j}}-a\right|>\tau$ occurs infinitely often as $m_{j} \rightarrow \infty$, for any $\tau>0$. By Borel Cantelli Lemma, this holds if $\sum_{m_{j}=1}^{\infty}\left\langle\mathbb{1}_{\left|M_{m_{j}}-a\right|>\tau}, \nu_{j}\right\rangle<\infty$. Moreover, independence of the $z_{i}$ 's imply that $\left\langle\mathbb{1}_{\left|M_{m_{j}}-a\right|>\tau}, \nu_{j}\right\rangle=\left\langle\mathbb{1}_{M_{m_{j}}<a-\tau}, \nu_{j}\right\rangle=$ $\left(\left\langle\mathbb{1}_{\epsilon_{k_{j}}\left(z_{i}\right)<a-\tau}, \nu_{j}\right\rangle\right)^{m_{j}}$. We observe that by definition of $a$, for any $\tau>0$, we always have $\left\langle\mathbb{1}_{\epsilon_{k_{j}}\left(z_{i}\right)<a-\tau}, \nu_{j}\right\rangle<1$. Therefore, $\sum_{m_{j}=1}^{\infty}\left\langle\mathbb{1}_{\left|M_{m_{j}}-a\right|>\tau}, \nu_{j}\right\rangle=\frac{1}{1-\left\langle\mathbb{1}_{\epsilon_{k_{j}}\left(z_{i}\right)<a-\tau}, \nu_{j}\right\rangle}$ is a convergent geometric series and we obtain the sought result. Finally, to show statement iii) we refer to the analysis used to establish the convergence of the standard CE method [2, Proposition 5.3]. The latter proposition relies on the following statement: under Assumption $\mathrm{B}$, assume a set $\hat{C} \subseteq \mathbb{R}^{p}$ converges almost surely as $m \rightarrow \infty$ towards $C \subseteq \mathbb{R}^{p}$; then almost surely we have that

$$
\lim _{m \rightarrow \infty} \underset{\nu^{\theta} \in \mathcal{V}}{\arg \max } \frac{1}{m} \sum_{i=1}^{m} \mathbb{1}_{\hat{C}\left(z_{i}\right)} \frac{\mu\left(z_{i}\right)}{\nu_{j-1}\left(z_{i}\right)} \ln \nu^{\theta}\left(z_{i}\right)=\underset{\nu^{\theta} \in \mathcal{V}}{\arg \max }\left\langle\mathbb{1}_{C} \frac{\mu}{\nu_{j-1}} \ln \nu^{\theta}, \nu_{j-1}\right\rangle,
$$

where $z_{i}$ 's are i.i.d. samples drawn according to $\nu_{j-1}$. Setting $\hat{C}=\hat{A}_{j}^{\left(k_{j-1}\right)}$ and $C=A_{j}^{\left(k_{j-1}\right)}$ and using the identity

$$
\underset{\nu^{\theta} \in \mathcal{V}}{\arg \max }\left\langle\mathbb{1}_{A_{j}^{\left(k_{j-1}\right)}} \frac{\mu}{\nu_{j-1}} \ln \nu^{\theta}, \nu_{j-1}\right\rangle=\underset{\nu^{\theta} \in \mathcal{V}}{\arg \max }\left\langle\mathbb{1}_{A_{j}^{\left(k_{j-1}\right)}} \ln \nu^{\theta}, \mu\right\rangle,
$$

we see that, to prove that approximation 4.3 converges almost surely towards the optimal solution (5.5), it is sufficient to show that the set $\hat{A}_{j}^{\left(k_{j-1}\right)}$ converges almost surely towards $A_{j}^{\left(k_{j-1}\right)}$. This is equivalent to show that $\gamma\left(\hat{\nu}_{j-1}, \rho_{j-1}, \phi^{\left(k_{j-1}\right)}\right)$ and $\alpha_{k_{j-1}}\left(\hat{\nu}_{j-1}\right)$ converge almost surely towards $\gamma\left(\nu_{j-1}, \rho_{j-1}, \phi^{\left(k_{j-1}\right)}\right)$ and $\alpha_{k_{j-1}}\left(\nu_{j-1}\right)$, which is precisely statement $i$ ) and $i i)$.

\section{Appendix D. Proof of the Second Statement of Theorem 5.1.}

Assuming that $\nu_{\hat{A}_{j}}^{\star} \in \mathcal{V}$ and $m_{j}$ large enough, the variance writes 


$$
\begin{aligned}
\mathbb{E}\left[\left(p_{A, \nu_{j}}^{I S}-p_{A}\right)^{2}\right] & =\frac{1}{m_{j}} \operatorname{var}\left(\mathbb{1}_{A} \frac{\mu}{\nu_{j}}, \nu_{j}\right)=\frac{1}{m_{j}}\left(\left\langle\left(\mathbb{1}_{A} \frac{\mu}{\nu_{j}}\right)^{2}, \nu_{j}\right\rangle-p_{A}^{2}\right) \\
& =\frac{1}{m_{j}}\left(\left\langle\frac{\mathbb{1}_{A} \mu}{\nu_{j}}, \mu\right\rangle-p_{A}^{2}\right)=\frac{1}{m_{j}}\left(p_{\hat{A}_{j}}\left\langle\frac{\mathbb{1}_{A}}{\mathbb{1}_{\hat{A}_{j}}}, \mu\right\rangle-p_{A}^{2}\right)=\frac{p_{A}}{m_{j}}\left(p_{\hat{A}_{j}}-p_{A}\right),
\end{aligned}
$$

where the second equality follows from the fact that estimator $(2.2)$ is unbiased, the fourth equality substitutes $\nu_{j}$ by the zero-variance density (2.4) because of the assumption $\nu_{\hat{A}_{j}}^{\star} \in \mathcal{V}$ and the asymptotic hypothesis, and the fifth equality is obtained using the condition $\hat{A}_{j} \supseteq A$ fulfilled by the $\hat{A}_{j}$ 's.

\section{Acknowledgements}

The author wishes to thank Frédéric Cérou and Mathias Rousset for fruit-full discussions on Monte-Carlo approximations and rare event simulation. He also thanks anonymous referees for their insightful comments which helped in improving the paper. Finally, he is sincerely grateful to B.L. for providing him a great deal of understanding.

\section{REFERENCES}

[1] Reuven Y Rubinstein, "Optimization of computer simulation models with rare events," European J. of Operational Research, vol. 99, no. 1, pp. 89-112, 1997.

[2] Tito Homem de Mello and Reuven Y. Rubinstein, "Rare event estimation for static models via cross-entropy and importance sampling," 2002.

[3] Reuven Y Rubinstein and Dirk P Kroese, Simulation and the Monte Carlo method, vol. 10, John Wiley \& Sons, 2016.

[4] Pieter-Tjerk De Boer, Dirk P Kroese, Shie Mannor, and Reuven Y Rubinstein, "A tutorial on the cross-entropy method," Annals of operations research, vol. 134, no. 1, pp. 19-67, 2005.

[5] Barron J Bichon, John M McFarland, and Sankaran Mahadevan, "Efficient surrogate models for reliability analysis of systems with multiple failure modes," Reliability Engineering \& System Safety, vol. 96, no. 10, pp. 1386-1395, 2011.

[6] Vincent Dubourg, Bruno Sudret, and Franois Deheeger, "Metamodel-based importance sampling for structural reliability analysis," Probabilistic Engineering Mechanics, vol. 33, pp. 47-57, 2013.

[7] Benjamin Peherstorfer, Tiangang Cui, Youssef Marzouk, and Karen Willcox, "Multifidelity importance sampling," Computer Methods in Applied Mechanics and Engineering, vol. 300, pp. $490-509,2016$.

[8] Benjamin Peherstorfer and Karen Willcox, "Online adaptive model reduction for nonlinear systems via low-rank updates," SIAM J. on Scientific Computing, vol. 37, no. 4, pp. A2123-A2150, 2015.

[9] Keyi Wu and Jinglai Li, "A surrogate accelerated multicanonical monte carlo method for uncertainty quantification," J. of Computational Physics, vol. 321, pp. 1098-1109, 2016.

[10] Vissarion Papadopoulos, Dimitris G Giovanis, Nikos D Lagaros, and Manolis Papadrakakis, "Accelerated subset simulation with neural networks for reliability analysis," Computer Methods in Applied Mechanics and Engineering, vol. 223, pp. 70-80, 2012.

[11] Elisabeth Ullmann and Iason Papaioannou, "Multilevel estimation of rare events," SIAM/ASA J. on Uncertainty Quantification, vol. 3, no. 1, pp. 922-953, 2015.

[12] Benjamin Peherstorfer, Boris Kramer, and Karen Willcox, "Multifidelity preconditioning of the cross-entropy method for rare event simulation and failure probability estimation," SIAM/ASA Journal of Uncertainty Quantification, 2017.

[13] Jing Li, Jinglai Li, and Dongbin Xiu, "An efficient surrogate-based method for computing rare failure probability," J. of Computational Physics, vol. 230, no. 24, pp. 8683 - 8697, 2011.

[14] Peng Chen and Alfio Quarteroni, "Accurate and efficient evaluation of failure probability for partial different equations with random input data," Computer Methods In Applied Mechanics And Engineering, vol. 267, pp. 28. 233-260, 2013.

[15] Alfio Quarteroni, Andrea Manzoni, and Federico Negri, Reduced basis methods for partial differential equations: an introduction, vol. 92, Springer, 2015. 
[16] Siu-Kui Au and James Beck, "Important sampling in high dimensions," Structural safety, vol. 25, no. 2, pp. 139-163, 2003.

[17] Patrick Héas, Frédéric Lavancier, and Souleymane Kadri-Harouna, "Self-similar prior and wavelet bases for hidden incompressible turbulent motion," SIAM J. on Imaging Sciences, vol. 7, no. 2, pp. 1171-1209, 2014.

[18] Allan Gut, Probability: A Graduate Course, Springer Texts in Statistics. New York, 2006. 